Research Article

\title{
Chaotic Convection in a Viscoelastic Fluid Saturated Porous Medium with a Heat Source
}

\author{
B. S. Bhadauria \\ Department of Mathematics, Institute of Science, Banaras Hindu University, Varanasi 221005, India \\ Correspondence should be addressed to B. S. Bhadauria; mathsbsb@yahoo.com
}

Received 12 October 2015; Revised 5 January 2016; Accepted 28 January 2016

Academic Editor: Nasser-Eddine Tatar

Copyright (C) 2016 B. S. Bhadauria. This is an open access article distributed under the Creative Commons Attribution License, which permits unrestricted use, distribution, and reproduction in any medium, provided the original work is properly cited.

Chaotic convection in a viscoelastic fluid saturated porous layer, heated from below, is studied by using Oldroyd's type constituting relation and in the presence of an internal heat source. A modified Darcy law is used in the momentum equation, and a heat source term has been considered in energy equation. An autonomous system of fourth-order differential equations has been deduced by using a truncated Fourier series. Effect of internal heat generation on chaotic convection has been investigated. The asymptotic behavior can be stationary, periodic, or chaotic, depending upon the flow parameters. Construction of four-scroll, or "two-butterfly," and chaotic attractor has been examined.

\section{Introduction}

Transport phenomenon in a fluid saturated porous medium is of great practical importance in many areas such as geothermal energy utilization, oil reservoirs, solar energy storage systems, passive cooling of nuclear reactors, pollutant transport in ground water, and storage of chemical and agricultural products, to mention a few. The problem of convection in a fluid saturated porous medium has been studied during the past few decades due to its applications in thermal and engineering sciences. An interesting problem was studied by Horton and Rogers [1] and independently by Lapwood [2], who addressed the Rayleigh-Bénard convection in porous media. Katto and Masuoka [3] employed Darcy's law to express the fluid characteristics in porous layer and experimentally showed the effect of Darcy's number on the onset conditions of buoyancy-driven convection. Important reviews of most of the findings on convection in porous medium are given by Ingham and Pop [4], Vafai [5], and Nield and Bejan [6].

The concept of chaos was first introduced by Poincaré [7, 8], who investigated orbits in celestial mechanics and realized that the dynamical system generated by the three-body problem is quite sensitive to the initial conditions exhibiting chaotic behavior. Since the introduction of the chaotic attractors by Lorenz [9] to study atmospheric convection, many chaotic systems have been introduced, such as Rössler [10], Chen and Ueta [11] systems. Because of their potential applications in engineering, the study of chaotic systems has attracted the interest of many researchers. Recently Vadász et al. [12] have investigated the effect of vertical vibrations on chaotic convection in porous medium employing Darcy model. Their results show that periodic solutions and chaotic solutions alternate as the value of the scaled Rayleigh number varies, when forced vibrations are present. Very recently Kiran and Bhadauria [13] have studied chaotic convection in a Newtonian fluid saturated porous medium under temperature modulation at the boundaries. They found that the effect of temperature modulation is to enhance the behavior of chaotic motion. Very recently Bhadauria et al. [14] and Bhadauria and Kiran [15] have studied the chaotic convection using different models.

Although viscoelastic fluid in porous media has been considered many years before by Marshall and Metzner [16] and James and McLaren [17], it is only recently that attention has been given to convection in viscoelastic fluid saturated porous media. Kim et al. [18] studied thermal instability of viscoelastic fluid in porous media by making linear and nonlinear stability analyses and obtained the stability criteria 
for convective flow. Later on Yoon et al. [19] studied the onset of oscillatory convection in a horizontal porous layer saturated with viscoelastic fluid by using linear theory. The linear stability of a viscoelastic fluid saturated densely packed horizontal porous layer heated from below and cooled from above was investigated by Malashetty et al. [20], using the Oldroyd-B type fluid. Since linear stability analysis cannot provide the information about the values of convection amplitudes nor about the aperiodic or chaotic motions, nonlinear analysis has to be performed. Kumar and Bhadauria [21-23] studied nonlinear double diffusive convection in a rotating porous layer saturated with viscoelastic fluid. Recently Bhadauria and Kiran [24, 25] studied oscillatory nonlinear thermal instability in viscoelastic fluids under the effect of temperature and gravity modulations.

Lorenz [9] studied nonlinear analysis of Rayleigh-Bénard convection in a fluid layer considering a truncated representation of Fourier series involving only two terms. Later on Khayat [26-28] and Abu-Ramadan et al. [29] followed Lorenz's work to derive the fourth-order system in Maxwell fluid and Oldroyd's fluid layers. Akhatov and Chemberisova [30] studied Rayleigh-Bénard convection in Oldroyd's fluid saturated porous layer with negligible Darcy effect and deduced a three-order autonomous chaotic system. Chaotic Rayleigh-Bénard convection in Oldroyd's fluid saturated porous medium using a thermal equilibrium model was investigated by Sheu et al. [31]. Recently Bhadauria and Kiran [15] studied double diffusive chaotic and oscillatory magnetoconvection in a viscoelastic fluid under G-jitter and found that modulated gravity field can be used either to delay or enhance the heat and mass transfer in the system.

Further, there can be a large number of practically important situations, where the porous material offers its own source of heat, thus setting up the convective flow in a different way, through the local heat generation within the porous media. This situation may occur through radioactive decay or through, in the present perspective, a relatively weak exothermic reaction which can take place within the porous material. It is well known that internal heating is the main source of energy for celestial bodies, which keeps the celestial objects warm and active. It is due to the internal heating of the earth that there exists a thermal gradient between the interior and the exterior of the earth's crust, saturated by multicomponents fluids, which helps convective flow, thereby transferring the thermal energy towards the surface of the earth. Therefore, the role of internal heat generation becomes very important in several applications that include geophysics, reactor safety analyses, metal waste form development for spent nuclear fuel, fire and combustion studies, and storage of radioactive materials. However, there are relatively very few studies avaliable in which the effect of internal heating on convective flow has been investigated. Some of these studies are Bhattacharyya and Jena [32], Haajizadeh et al. [33], Rionero and Straughan [34], Rao and Wang [35], Parthiban and Patil [36], Herron [37], Khalili and Huettel [38], Joshi et al. [39], Bhadauria et al. [40], Bhadauria [41], and Bhadauria et al. [42].
The motive for the present work is to study the effect of internal heat source on dynamics of convection in a viscoelastic fluid saturated porous medium. In the momentum equation, the viscoelastic model of the Oldroyd type is considered by a modified Darcy law. To deduce fourdimensional system, the Fourier series expansion has been applied to the governing equations of the thermal convection in a viscoelastic fluid saturated porous medium. The effects of internal heat source, relaxation, and retardation parameters on the dynamics of the system have been examined in detail. Further, the present system has been reduced to some famous systems provided in the literature.

\section{Mathematical Formulation}

An infinitely extended horizontal viscoelastic fluid saturated porous layer, confined between two impermeable boundaries at $z=0$ and $z=d$, heated from below and cooled from above, has been considered. A Cartesian frame of reference is chosen in such a way that the origin lies on the lower plane and the $z$-axis as vertical upward. Adverse temperature gradient is applied across the porous layer and the lower and upper planes are kept at temperatures $T_{0}+\Delta T$ and $T_{0}$, respectively. Oberbeck-Boussinesq approximation is applied to account the effect of density variations. Under these postulates the governing equations for thermal convection in a viscoelastic fluid saturated porous medium are given by

$$
\begin{aligned}
& \nabla \cdot \mathbf{q}=0 \\
& \left(1+\lambda_{1} \frac{\partial}{\partial t}\right)\left[\frac{\rho_{0}}{\phi} \frac{\partial \mathbf{q}}{\partial t}+\nabla p+\rho \mathbf{g}\right]+\frac{\mu}{K}\left(1+\lambda_{2} \frac{\partial}{\partial t}\right) \mathbf{q} \\
& \quad=0 \\
& \left(\frac{\partial}{\partial t}+\mathbf{q} \cdot \nabla\right) T=\kappa_{T} \nabla^{2} T+Q\left(T-T_{0}\right), \\
& \rho=\rho_{0}\left[1-\alpha_{T}\left(T-T_{0}\right)\right],
\end{aligned}
$$

where $\mathbf{q}$ is velocity $(u, v, w), \mu$ is the dynamic viscosity, $K$ is permeability, $\kappa_{T}$ is the thermal diffusivity, $T$ is temperature, $\alpha_{T}$ is thermal expansion coefficient, and $\rho$ is the density, while $\rho_{0}$ and $T_{0}$ are the reference density and temperature, respectively.

The externally imposed thermal boundary conditions are given by

$$
\begin{aligned}
& T=T_{0}+\Delta T, \quad \text { at } z=0, \\
& T=T_{0}, \quad \text { at } z=d,
\end{aligned}
$$

where $\Delta T$ is the temperature difference across the porous medium. 


\section{Basic State}

The basic state is assumed to be quiescent, and the quantities in this state are given by

$$
\begin{aligned}
\mathbf{q}_{b} & =0, \\
p & =p_{b}(z), \\
T & =T_{b}(z), \\
\rho & =\rho_{b}(z) .
\end{aligned}
$$

Substituting (6) in (1)-(4), we get the following relations, which helps us to define basic state pressure and temperature:

$$
\begin{aligned}
\frac{d p_{b}}{d z} & =-\rho_{b} g \\
\kappa_{T} \frac{d^{2}\left(T_{b}-T_{0}\right)}{d z^{2}}+Q\left(T_{b}-T_{0}\right) & =0 \\
\rho_{b} & =\rho_{0}\left[1-\alpha_{T}\left(T_{b}-T_{0}\right)\right] .
\end{aligned}
$$

The solution of (8), subject to the boundary conditions (5), is given by

$$
T_{b}=T_{0}+\Delta T \frac{\sin \sqrt{\left(Q / \kappa_{T}\right)}(1-z / d)}{\sin \sqrt{\left(Q / \kappa_{T}\right)}}
$$

The finite amplitude perturbations on the basic state are superposed in the following form:

$$
\begin{aligned}
& \mathbf{q}=\mathbf{q}_{b}+\mathbf{q}^{\prime}, \\
& \rho=\rho_{b}+\rho^{\prime}, \\
& p=p_{b}+p^{\prime}, \\
& T=T_{b}+T^{\prime} .
\end{aligned}
$$

We introduce (11) and the basic state temperature field given by (10) in (1)-(4). The resulting equations are then nondimensionalized using the following transformations:

$$
\begin{aligned}
(x, y, z) & =\left(x^{*}, y^{*}, z^{*}\right) d, \\
t & =\frac{d^{2}}{\kappa_{T}} t^{*}, \\
\mathbf{q}^{\prime} & =\frac{\kappa_{T}}{d} q^{*}, \\
T^{\prime} & =(\Delta T) T^{*}, \\
p^{\prime} & =\frac{\mu \kappa_{T}}{K} p^{*}, \\
\lambda_{1} & =\frac{d^{2}}{\kappa_{T}} \lambda_{1}^{*}, \\
\lambda_{2} & =\frac{d^{2}}{\kappa_{T}} \lambda_{2}^{*} .
\end{aligned}
$$

Introducing the stream function $\psi$ such that $u^{*}=-\partial \psi / \partial z^{*}$ and $w^{*}=\partial \psi / \partial x^{*}$ in the resulting momentum equation (1) and then eliminating the pressure $p$ by operating curl on it, we get the following momentum and energy equations in nondimensionalized form (dropping the asterisks) as

$$
\begin{aligned}
& {\left[\frac{1}{\mathrm{Va}}\left(1+\lambda_{1} \frac{\partial}{\partial t}\right) \frac{\partial}{\partial t}+\left(1+\lambda_{2} \frac{\partial}{\partial t}\right)\right]\left(\frac{\partial^{2} \psi}{\partial x^{2}}+\frac{\partial^{2} \psi}{\partial z^{2}}\right)} \\
& \quad=-\operatorname{Ra}\left(1+\lambda_{1} \frac{\partial}{\partial t}\right) \frac{\partial T}{\partial x}, \\
& \frac{\partial T}{\partial t}-\frac{\partial \psi}{\partial x} \frac{\partial T}{\partial z}+\frac{\partial \psi}{\partial z} \frac{\partial T}{\partial x}-R_{i} T \\
& \quad=\left(\frac{\partial^{2}}{\partial x^{2}}+\frac{\partial^{2}}{\partial z^{2}}\right) T+\frac{\partial \psi}{\partial x} \frac{d T_{b}}{d z}
\end{aligned}
$$

where $\mathrm{Va}=\phi \operatorname{Pr} / \mathrm{Da}$ is the Vadasz number, $\mathrm{Ra}=$ $\alpha_{T} g K(\Delta T) d / \nu \kappa_{T}$ is the Rayleigh number, $R_{i}=Q d^{2} / \kappa_{T}$ is the internal Rayleigh number, $\mathrm{Da}=K / d^{2}$ is the Darcy number, and $\operatorname{Pr}=\nu / \kappa_{T}$ is the Prandtl number.

The nondimensional basic temperature field $T_{b}$, which appears in (14), can be obtained from the expression (10) as

$$
\frac{d T_{b}}{d z}=-\frac{\sqrt{R_{i}} \cos \sqrt{R_{i}}(1-z)}{\sin \sqrt{R_{i}}} .
$$

\section{Mathematical Solution}

To obtain the solution of the nonlinear coupled system of partial differential equations (13)-(14), we represent the stream function and temperature in the following Fourier series expressions $[12,13,15]$ :

$$
\begin{aligned}
& \psi=A_{11}(t) \sin (a x) \sin (\pi z), \\
& T=B_{11}(t) \cos (a x) \sin (\pi z)+B_{02}(t) \sin (2 \pi z) .
\end{aligned}
$$

Substituting expressions (16) in (13)-(14), using the orthogonality condition with the eigenfunctions associated with expressions (16), and integrating over the domain, we get a set of ordinary differential equations for the time evolution of the amplitudes, in the form

$$
\begin{aligned}
& \delta^{2}\left[\left(1+\Gamma \frac{\partial}{\partial \tau}\right)+\operatorname{Va}\left(1+\Gamma \Lambda \frac{\partial}{\partial \tau}\right)\right] A_{11}(\tau) \\
& =a \operatorname{VaRa}\left(1+\Gamma \frac{\partial}{\partial \tau}\right) B_{11}(\tau) \\
& \frac{d B_{11}(\tau)}{d \tau} \\
& =2 a F A_{11}(\tau)+\left(R_{i}-\delta^{2}\right) B_{11}(\tau) \\
& \quad-\pi a A_{11}(\tau) B_{02}(\tau) \\
& \frac{d B_{02}(\tau)}{d \tau}=\left(R_{i}-4 \pi^{2}\right) B_{02}(\tau)+\frac{\pi a}{2} A_{11}(\tau) B_{11}(\tau)
\end{aligned}
$$


where $\Gamma$ is nondimensional relaxation time, $\Lambda$ is ratio of retardation time to relaxation time, and $\delta^{2}=\left(\pi^{2}+a^{2}\right)$. Also

$$
F=-\frac{\sqrt{R_{i}}}{\sin \sqrt{R_{i}}} \int_{0}^{1} \cos \sqrt{R_{i}}(1-z) \sin ^{2} \pi z d z .
$$

In (17) above, time $t$ has been rescaled as $\tau=\left(\pi^{2}+a^{2}\right) t$. The above low-order spectral model may qualitatively reproduce convective phenomenon observed in the full system. Further, the solution of the system can be used as initial values in studying the fully nonlinear convection problem.

Now, for our convenience, we use the following notations:

$$
\begin{aligned}
& R=\frac{a^{2} \mathrm{Ra}}{\delta^{4}}, \\
& \sigma=\frac{\mathrm{Va}}{\delta^{2}}, \\
& b=\frac{R_{i}-\delta^{2}}{\delta^{2}}, \\
& c=\frac{R_{i}-4 \pi^{2}}{\delta^{2}}, \\
& d=2 R F .
\end{aligned}
$$

Then after rescaling the amplitudes in the form

$$
\begin{aligned}
& X=\frac{\pi a A_{11}}{\delta^{2} \sqrt{2}}, \\
& Y=\frac{\pi R B_{11}}{\sqrt{2}}, \\
& Z=-\pi R B_{02}
\end{aligned}
$$

we get the following set of equations:

$$
\begin{aligned}
\dot{X} & =W, \\
\dot{Y} & =-d X+b Y-X Z, \\
\dot{Z} & =c Z+X Y, \\
\dot{W} & =\sigma\left[-\left(d+\Gamma^{-1}\right) X+\left(b+\Gamma^{-1}\right) Y-X Z\right. \\
& \left.-\left(\Lambda+\frac{1}{\sigma \Gamma}\right) W\right],
\end{aligned}
$$

where "." denotes the derivative with respect to the scaled time $\tau$.

\section{Some Special Cases}

(1) For noninternal heat generation case, we have $R_{i} \rightarrow 0$; then $d \rightarrow-R$ or $F \rightarrow-1 / 2, b=-1$, and $c=-4 \gamma$, where $\gamma=\pi^{2} /\left(\pi^{2}+a^{2}\right)$, and we get

$$
\begin{aligned}
& \dot{X}=W, \\
& \dot{Y}=R X-Y-X Z,
\end{aligned}
$$

$$
\begin{aligned}
\dot{Z} & =X Y-4 \gamma Z \\
\dot{W} & =\sigma\left[\left(R-\Gamma^{-1}\right) X+\left(\Gamma^{-1}-1\right) Y-X Z\right. \\
& \left.-\left(\Lambda+\frac{1}{\sigma \Gamma}\right) W\right]
\end{aligned}
$$

which is similar to Sheu et al. [31] though with different coefficients.

(2) If we let

$$
P=Y-\Lambda X-\frac{W}{\sigma}
$$

then the above system takes the form

$$
\begin{aligned}
& \dot{X}=\sigma(Y-\Lambda X-P), \\
& \dot{Y}=R X-Y-X Z, \\
& \dot{Z}=X Y-4 \gamma Z, \\
& \dot{P}=\Gamma^{-1}[(1-\Lambda) X-P]
\end{aligned}
$$

which is identical to Khayat's [26-28] system with the assumption of the parameters: $\sigma \rightarrow \operatorname{Pr}, R \rightarrow r, \Lambda \rightarrow$ $\alpha R \nu, \Gamma^{-1} \rightarrow \delta$, and $4 \gamma \rightarrow b$.

(3) If, in system (21), we consider the case of Newtonian fluid by taking $\Gamma \rightarrow 0, \Lambda \rightarrow 0$, we obtain

$$
\begin{aligned}
& \dot{X}=\sigma(Y-X), \\
& \dot{Y}=-d X+b Y-X Z, \\
& \dot{Z}=X Y+c Z .
\end{aligned}
$$

(4) If, in the above system (25), we consider $R_{i} \rightarrow 0$, then we get $d \rightarrow-R$ or $F(z) \rightarrow-1 / 2, b=-1$, and $c=-4 \gamma$, where $\gamma=\pi^{2} /\left(\pi^{2}+a^{2}\right)$,

$$
\begin{aligned}
& \dot{X}=\sigma(Y-X), \\
& \dot{Y}=R X-Y-X Z, \\
& \dot{Z}=X Y-4 \gamma Z
\end{aligned}
$$

which are famous Lorenz [9] equations.

(5) In the limit case $\sigma \rightarrow \infty$, that is, Darcy number $\mathrm{Da} \rightarrow$ 0 , the above system (26) reduces to

$$
\begin{aligned}
\dot{X} & =\frac{1}{\Lambda}\left[\left(R-\Gamma^{-1}\right) X+\left(\Gamma^{-1}-1\right) Y-X Z\right] \\
& =-\frac{\lambda_{1}}{\lambda_{2}} X Z-\frac{1-R \lambda_{1}}{\lambda_{2}} X+\frac{1-\lambda_{1}}{\lambda_{2}} Y, \\
\dot{Y} & =R X-Y-X Z, \\
\dot{Z} & =X Y-4 \gamma
\end{aligned}
$$

which is identical to the system given by Akhatov and Chembarisova [30]. 


\section{Stability Analysis}

5.1. Equilibrium Points. Setting the time derivatives of system (21) to vanish, we obtain the equilibrium points for velocity and temperature fields as

$$
\begin{aligned}
& W=0, \\
& d X-b Y+X Z=0, \\
& c Z+X Y=0, \\
& \sigma\left[-\left(d+\Gamma^{-1}\right) X+\left(b+\Gamma^{-1}\right) Y-X Z\right. \\
& \left.\quad-\left(\Lambda+\frac{1}{\sigma \Gamma}\right) W\right]=0 .
\end{aligned}
$$

Solving the above algebraic equations, we get the trivial solution

$$
X_{1}=Y_{1}=Z_{1}=W_{1}=0,
$$

which corresponds to pure heat conduction solution. This is known to be a possible solution though it is unstable when $R(\mathrm{Ra})$ is sufficiently large. The other two equilibrium points are

$$
\begin{aligned}
& {\left[X_{2,3}, Y_{2,3}, Z_{2,3}, W_{2,3}\right]} \\
& \quad=[ \pm \sqrt{c(d-b)}, \pm \sqrt{c(d-b)},(b-d), 0] ;
\end{aligned}
$$

the solutions $\left(X_{2,3}, Y_{2,3}, Z_{2,3}, W_{2,3}\right)$ characterize the onset of finite amplitude steady motions, where $R>b / a F$.

5.2. Stability of the Equilibrium Points. The Jacobian matrix of system (21) may be written as

$$
=\left[\begin{array}{cccc}
0 & 0 & 0 & 1 \\
-(d+Z) & b & -X & 0 \\
Y & X & c & 0 \\
\sigma\left[-\left(d+\Gamma^{-1}\right)-Z\right] & \sigma\left(b+\Gamma^{-1}\right) & -\sigma X & -\sigma \Lambda+\Gamma^{-1}
\end{array}\right] .
$$

The stability of the fixed point corresponding to pure conduction solution $\left(X_{1}=Y_{1}=Z_{1}=W_{1}=0\right)$ is governed by the roots of the following characteristic polynomial equation for the eigenvalues, $\lambda_{n}(n=1-4)$ :

$$
\begin{gathered}
(c-\lambda)\left[\lambda^{3}+\left\{\left(\sigma \Lambda+\Gamma^{-1}\right)-b\right\} \lambda^{2}+\sigma\left(d+\Gamma^{-1}\right)\right. \\
\left.-b\left(\sigma \Lambda+\Gamma^{-1}\right) \lambda+\sigma\left\{2 b d+(b+d) \Gamma^{-1}\right\}\right]=0 .
\end{gathered}
$$

Stability depends upon the value of $\Gamma$; for $\Gamma<(\sigma-$ $b) /[\sigma b(\Lambda-1)]$ there is an exchange of stability, and for other two steady state solutions origin loses its stability. When $\Gamma>$ $(\sigma-b) /[\sigma b(\Lambda-1)]$, there is a pair of pure imaginary roots of (32). The oscillatory or overstable solutions arise at a critical value of Rayleigh number given by

$$
\begin{aligned}
& R^{\text {osc }} \\
& =\frac{-\delta^{4}(1+\sigma \Lambda \Gamma)\left[b(1+\sigma \Lambda \Gamma)-\sigma(1+b \Gamma)-b^{2} \Gamma^{2}(\sigma \Lambda-b)\right]}{2 a^{2} \sigma F(z)(1+b \Gamma)(\sigma \Lambda-b)} .
\end{aligned}
$$

The stability of the fixed point corresponding to convection solution $(X=Y= \pm \sqrt{c(d-b)}, Z=(b-d), W=$ $0)$ is governed by the roots of the following characteristic polynomial equation for the eigenvalues, $\lambda_{n}(n=1-4)$ :

$$
\begin{aligned}
\lambda^{4} & +\left[\left(\sigma \Lambda+\Gamma^{-1}\right)-(b+c)\right] \lambda^{3}+[c d \\
& \left.-(b+c)\left(\sigma \Lambda+\Gamma^{-1}\right)+\sigma\left(b+\Gamma^{-1}\right)\right] \lambda^{2} \\
& +\left[c d\left(\sigma \Lambda+\Gamma^{-1}\right)-\sigma\left(b+\Gamma^{-1}\right)(2 b+c)\right. \\
& +\sigma c(d-b)] \lambda+2 \sigma b c\left(b+\Gamma^{-1}\right)=0 .
\end{aligned}
$$

The steady state solutions are useful because they predict that a finite amplitude solution to the system is possible for subcritical values of the Rayleigh number and that the minimum values of $\mathrm{Ra}$ for which a steady solution is possible lie below the critical values for instability to either a marginal state or an overstable infinitesimal perturbation.

\section{Results and Discussions}

In the previous section, the set of results were obtained for supercritical values of $R$ with the effect of internal heat generation. All the calculations were taken over using Mathematica's inbuilt forth-order Runge-Kutta method. Solutions were obtained using the same initial conditions which were selected to be in the neighbourhood of the positive convective fixed point. The common initial conditions, $X(0)=Y(0)=$ $Z(0)=0.9$ and $W(0)=0.1$, have been chosen. The time domain is taken as $0 \leq \tau \leq 100$ with a constant time step, $\Delta \tau=0.001$. In the paper, we demonstrate the effects of internal heat source and viscoelastic parameters on the system, in the form of space projections of trajectories onto the $Y-X, Z-X$, and $Z-Y$ planes, as the value of $R$ increases.

In Figure 1, the initial supercritical solutions are presented at fixed value of $R_{i}=0.1\left[1\left(a_{1}-a_{4}\right)\right], R_{i}=2\left[1\left(a_{5}-a_{8}\right)\right]$, and $R_{i}=5\left[1\left(a_{9}-a_{12}\right)\right]$ with $\Lambda=0.7$ and have been projected onto $Y$-X planes. From Figure 1, we observed that for weak internal heating, that is, $R_{i}=0.1$ at $R_{c}=0.987361$, the motionless solution loses its stability and the convection solution takes over. For $R=2$, trajectories move towards steady convection stability point on a straight line for a Rayleigh number slightly above the loss of stability of the motionless solution. From Figure 1, it is evident that there is phase (spiral) trajectory for $R=8$, as the flow exhibits an oscillatory decay. In Figure 1, spiralling approach of the trajectories towards the steady state fixed point is quite pronounced. This behavior is also inferred from the roots of (32). The flow undergoes a homoclinic bifurcation, as shown in Figure 1 for $R=9.294$, similar to that predicted by the Lorenz [9] equations. This is known as a global bifurcation which cannot be detected through local stability analysis around the fixed point. On further increasing the value of $R$, the flow becomes completely chaotic as can be depicted from the spatiotemporal structure of flow. The transition to chaos in this case is similar to that leading to the Lorenz attractor. It does not follow any of 

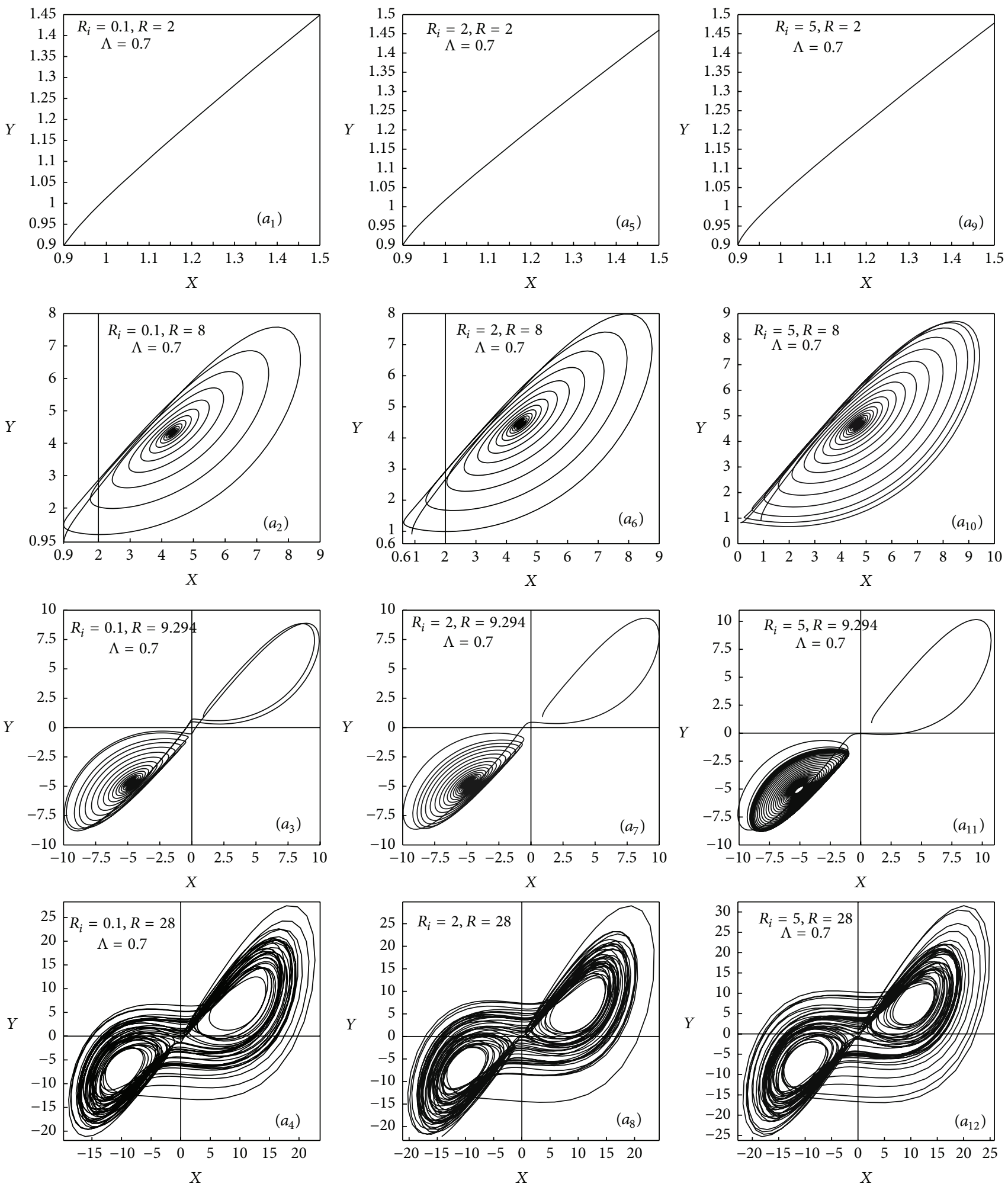

FIgURE 1: Projections and evolution of trajectories over the planes $X-Y$. 
the well known routes to chaos, such as through period doubling, quasiperiodicity, or intermittency [43]. The behavior of the system at $R=28$ is chaotic which is confirmed by the broadening of the base in the power spectrum. For the moderate heating, that is, $R_{i}=2$ at $R_{c}=0.756963$, the motionless solution loses its stability and the convection solution takes over. For $R=2$, trajectories move towards steady convection stability point on a straight line for a Rayleigh number slightly above the loss of stability of the motionless solution. At $R=8$, it shows the spiral approach before it is attracted towards steady state fixed point. As the value of $R$ increased to $R=$ 9.294, the flow becomes homoclinic in nature, and, on further increasing to $R=28$, a transition to chaos occurs. In the case of strong heating, for $R_{i}=5$, at $R_{c}=0.430905$, the motionless solution loses its stability and the convection solution takes over. For $R=2$, trajectories move towards steady convection stability point on a straight line for a Rayleigh number slightly above the loss of stability of the motionless solution. In this case for $R=8$ spiralling approach is even more pronounced, a fact which is consistent with the eigenvalues. From Figure 1 $\left(a_{11}\right)$, for $R=9.294$, a homoclinic bifurcation occurs which is more pronounced in this case. Finally at $R=28$, transition to chaos occurs. Figure $2\left(b_{1}-b_{12}\right)$ is the plots of $Y$-X plane at $R_{i}=0.1\left[2\left(b_{1}-b_{4}\right)\right], R_{i}=2\left[2\left(b_{5}-b_{8}\right)\right]$, and $R_{i}=5\left[2\left(b_{9}-b_{12}\right)\right]$ with $\Lambda=0.9$. In Figure $2\left(b_{1}-b_{12}\right)$, we find qualitatively similar results as shown in Figure $1\left(a_{1}-a_{12}\right)$, except that amplitudes take a little bit larger value than for $\Lambda=0.7$.

Figure 3 shows the initial supercritical solutions at fixed value of $R_{i}=0.1\left[1\left(c_{1}-c_{4}\right)\right], R_{i}=2\left[1\left(c_{5}-c_{8}\right)\right]$, and $R_{i}=$ $5\left[1\left(c_{9}-c_{12}\right)\right]$ with $\Lambda=0.7$, projected onto $Z$ - $X$ planes. From Figure $3\left(c_{1}-c_{12}\right)$ we observed that for weak heating, that is, $R_{i}=0.1$ at $R_{c}=0.987361$, the motionless solution loses stability and the convection solution takes over. For $R=2$ trajectories move towards steady convection stability point through a spiral for a Rayleigh number slightly above the loss of stability of the motionless solution. From Figure 3 $\left(c_{2}, c_{6}, c_{10}\right)$ it is clear that there is spiral trajectory for $R=8$ as the flow exhibits an oscillatory decay. In Figure $3\left(c_{2}, c_{6}, c_{10}\right)$, spiralling approach of the trajectories towards the steady state fixed point is more pronounced. For $R=9.19$, there is a homoclinic pattern of flow, as shown in Figure $3\left(c_{3}, c_{7}, c_{11}\right)$. Further increasing the value of $R$, there occurs a transition to chaotic convection as can be seen from Figure $3\left(c_{4}, c_{8}, c_{12}\right)$. The transition to chaos in this case is similar to that leading to the Lorenz attractor; one can easily see "two-butterfly" Figure $3\left(c_{4}\right)$ for $R=28$. For the case of moderate heating, that is, $R_{i}=2$ at $R_{c}=0.756963$, the motionless solution loses stability and the convection solution takes over. Trajectories move towards steady convection stability point through a spiral for a Rayleigh number slightly above the loss of stability of the motionless solution for $R=2$. At $R=8$ trajectories move via a spiral, before they are attracted to their steady state fixed points. At $R=9.19$, the flow becomes homoclinic in nature and attracted towards a steady state fixed point. On increasing the value of $R$ at $R=28$, a transition to chaos occurs and "two-butterfly" Figure $3\left(c_{4}\right)$ are obtained. For the case of strong heating, namely, $R_{i}=5$, the motionless solution loses its stability and the convection solution takes over at $R_{c}=0.430905$. For $R=2$ trajectories move towards steady convection stability point through a spiral for a Rayleigh number slightly above the loss of stability of the motionless solution. In this case for $R=8$, spiralling approach is even more pronounced, a fact which is consistent with the eigenvalues. From Figure $3\left(c_{11}\right)$ for $R=9.19$, a homoclinic bifurcation occurs which is more pronounced in this case. Finally at $R=28$ transition to chaos occurs and "twobutterfly" nature occurs. In Figure $4\left(d_{1}-d_{12}\right)$, which are for $\Lambda=0.9$, we found qualitatively similar results to those of Figure $3\left(c_{1}-c_{12}\right)$ with slightly large amplitudes.

The initial supercritical solutions, calculated at $R_{i}=0.1$, 2 , and 5 with $\Lambda=0.7$, projected onto $Z-Y$ planes have been presented in Figure 5. From Figure $5\left(e_{1}-e_{4}\right)$, we observe that for the case of weak heating, that is, at $R_{i}=0.1$, the motionless solution loses its stability at $R_{c}=0.987361$, and the convection solution takes over. For $R=2$ trajectories move towards steady convection stability point through a spiral for a Rayleigh number slightly above the loss of stability of the motionless solution. From Figure $5\left(e_{6}\right)$ it is clear that there are spiral trajectory for $R=8$ as the flow exhibits an oscillatory decay. In Figure $5\left(e_{5}\right)$, spiralical approach of the trajectories towards the steady state fixed point is more pronounced. For $R=9.45$, there is a homoclinic bifurcation, as shown in Figure $5\left(e_{7}\right)$. Further increasing the value of $R$, there occurs a transition to chaotic convection as can be seen from Figure $5\left(e_{8}\right)$. The transition to chaos in this case is similar to that leading to the Lorenz attractor, and one can easily see the transition to chaos for $R=28$. For the case of moderate heating, that is, $R_{i}=2$ at $R_{c}=0.756963$, the motionless solution loses its stability and the convection solution takes over; the trajectories move towards steady convection stability point through a spiral for a Rayleigh number slightly above the loss of stability of the motionless solution at $R=2$. At $R=8$ trajectories move via a spiral, before it is attracted to its steady state fixed point. At $R=9.45$, the flow becomes homoclinic in nature and attracted towards a steady state fixed point. On increasing the value of $R$ at $R=28$, a transition to chaos occurs. For the case of strong heating, namely, $R_{i}=5$ at $R_{c}=0.430905$, the motionless solution loses its stability and the convection solution takes over; for $R=2$ trajectories move towards steady convection stability point through a spiral for a Rayleigh number slightly above the loss of stability of the motionless solution. In this case for $R=8$, spiralling approach is even more pronounced, a fact which is consistent with the eigenvalues. From Figure 5 $\left(e_{11}\right)$ for $R=9.45$, a homoclinic bifurcation occurs which is more pronounced in this case. Finally at $R=28$ there occurs transition to chaos. Figure $6\left(f_{1}-f_{12}\right)$ is the plots of initial supercritical solutions, and it is found that the results are qualitatively similar to those obtained in Figure $5\left(e_{1}-e_{12}\right)$.

Figure 7 is the plots of three-dimensional chaotic bifurcation at different values of $R_{i}$ and $\Lambda$. Figures $8(\mathrm{a})-8(\mathrm{f})$ are the plots of time history of $X$ at different values of $R_{i}$, at $R=20$ and $R=28$ with initial points $(7,7,19,0)$ for $R_{i}=0.1$, $(7.3,7.3,20,0)$ for $R_{i}=2$, and $(7.6,7.6,21.9,0)$ for $R_{i}=5$. In Figures 9(a)-9(f), we plot the time history of $Y$ at different values of $R_{i}$, at $R=20$ and $R=28$ with initial points $(7,7,19,0)$ for $R_{i}=0.1,(7.3,7.3,20,0)$ for $R_{i}=2$, and $(7.6,7.6,21.9,0)$ for $R_{i}=5$. Figures $10(\mathrm{a})-10(\mathrm{f})$ are the plots of 

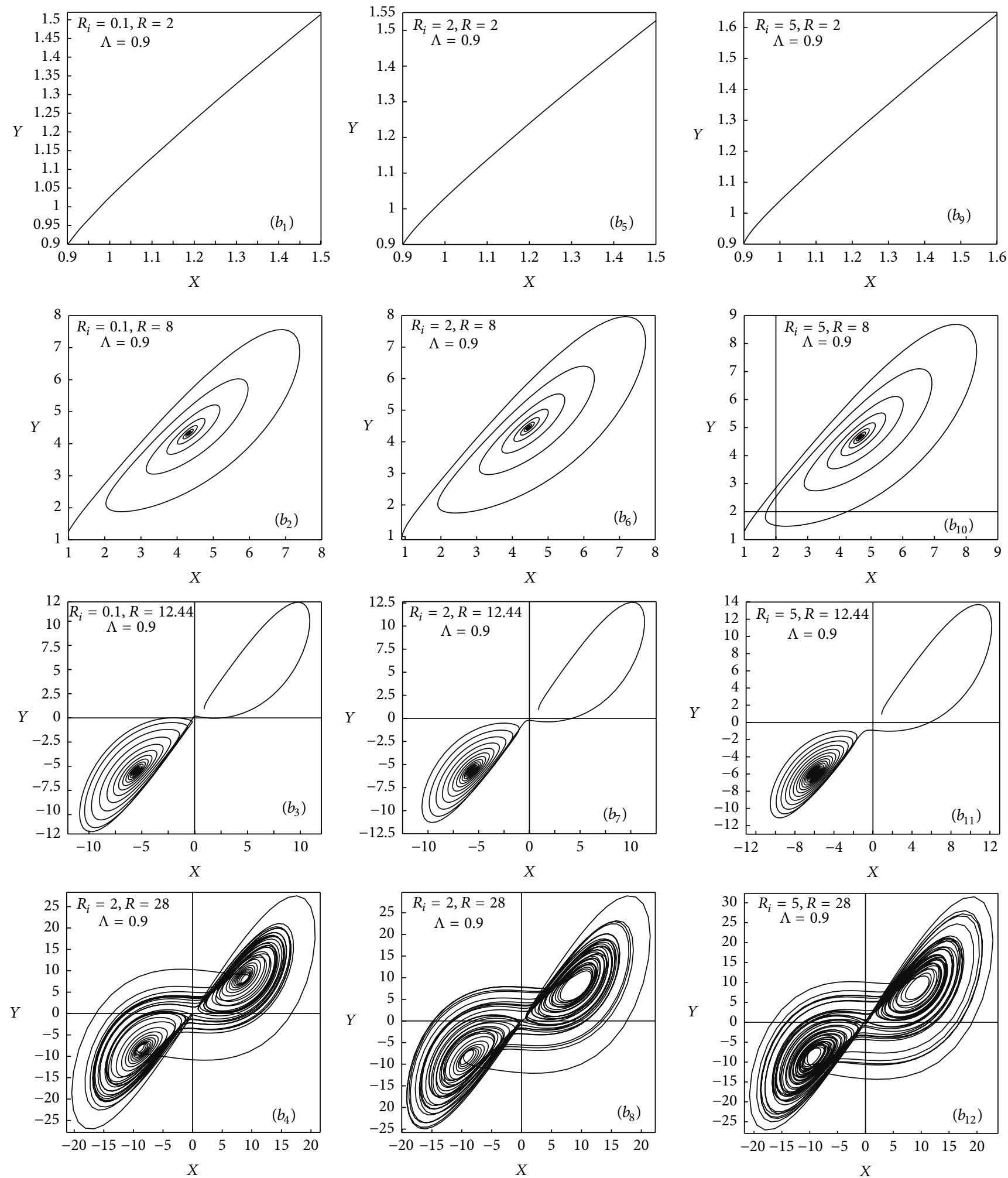

FIGURE 2: Projections and evolution of trajectories over the planes $X-Y$. 


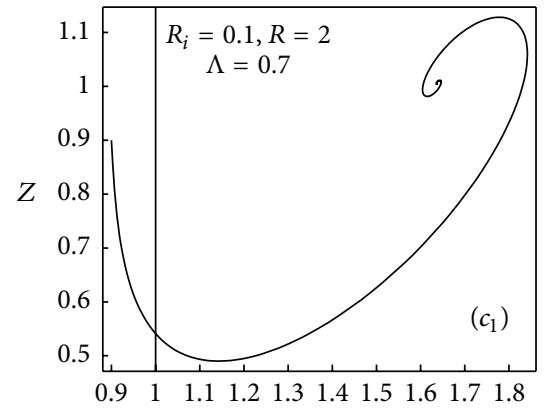

$X$
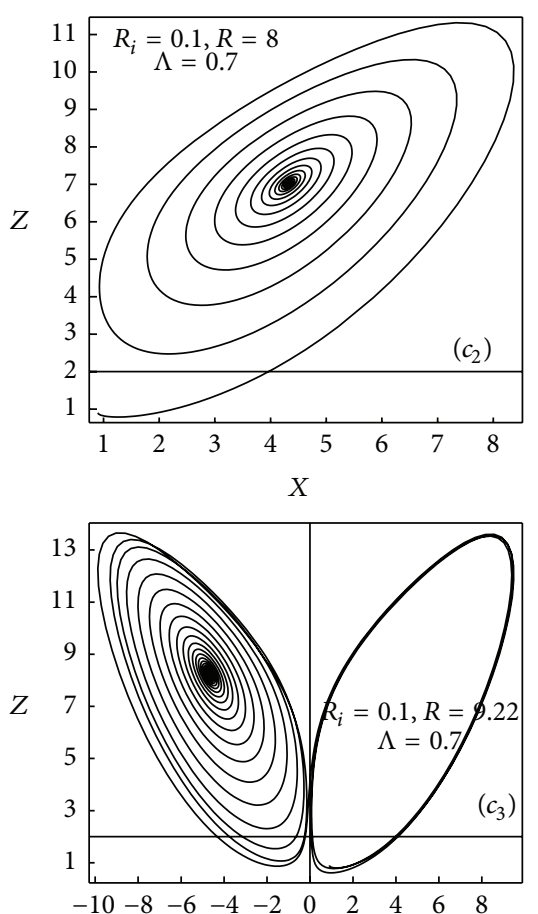

X

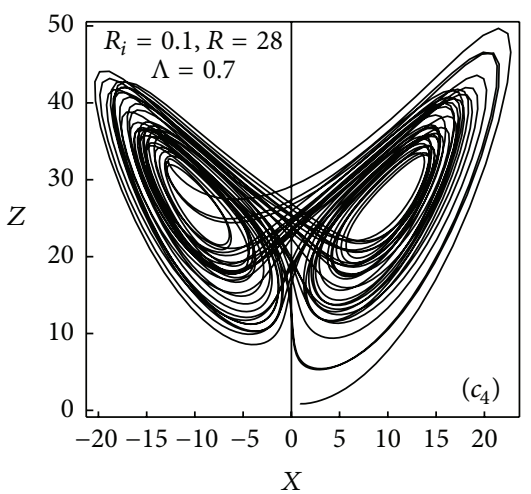

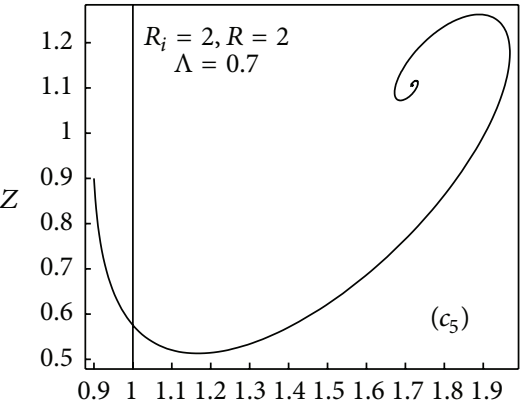

X
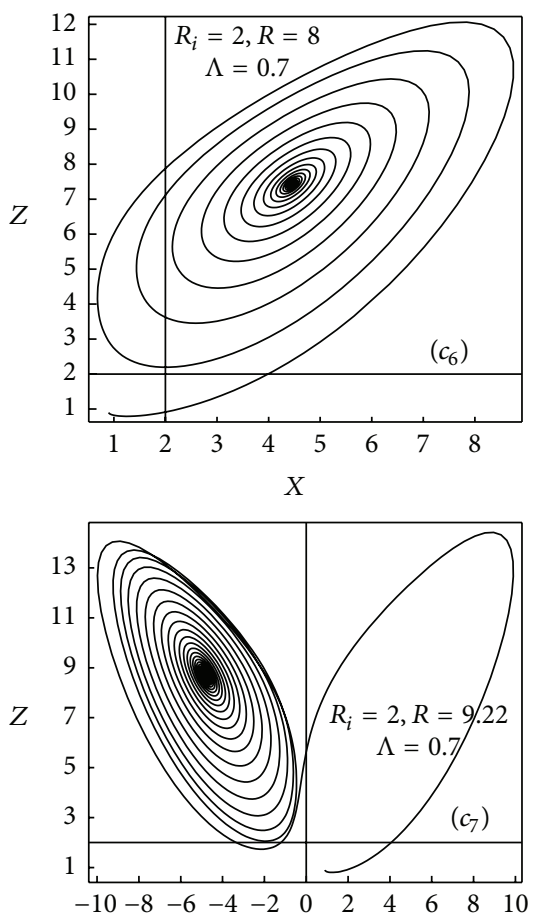

X

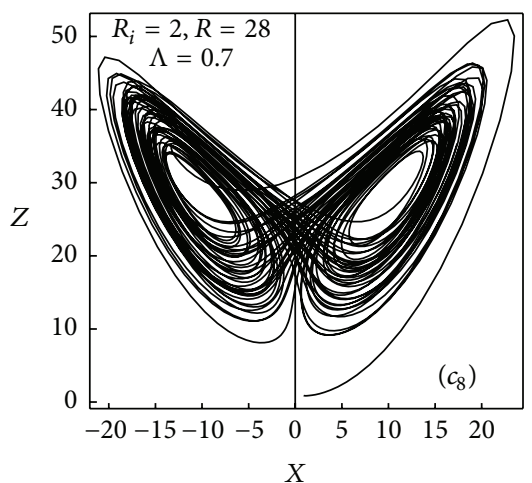

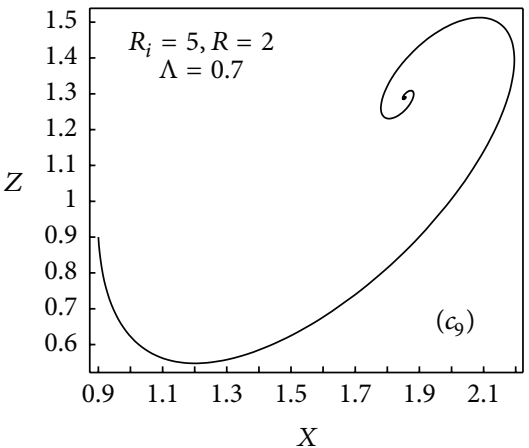
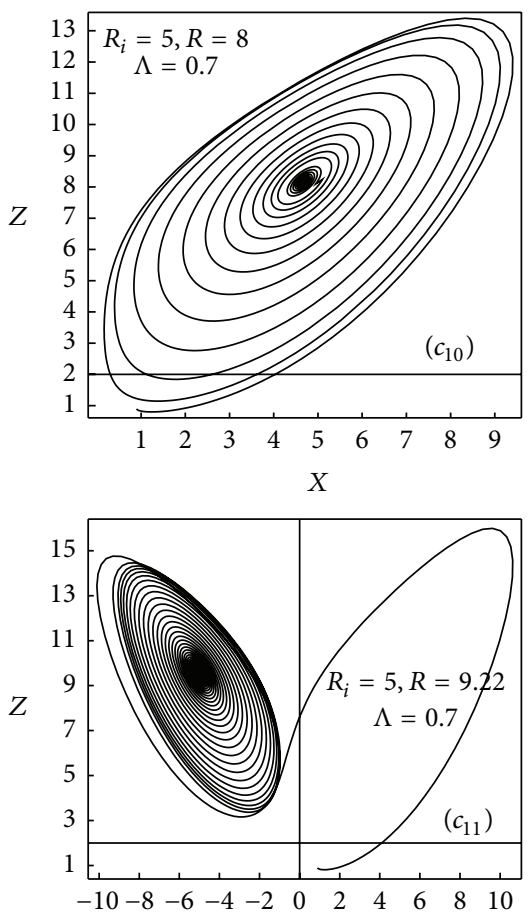

X

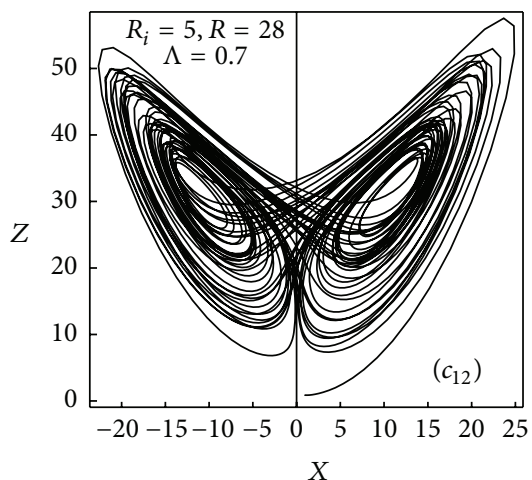

Figure 3: Projections and evolution of trajectories over the planes $X-Z$. 


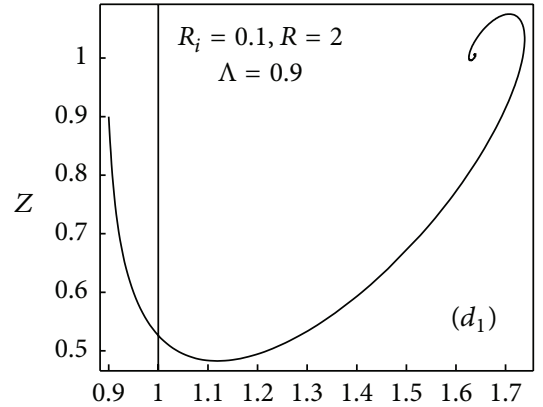

$X$
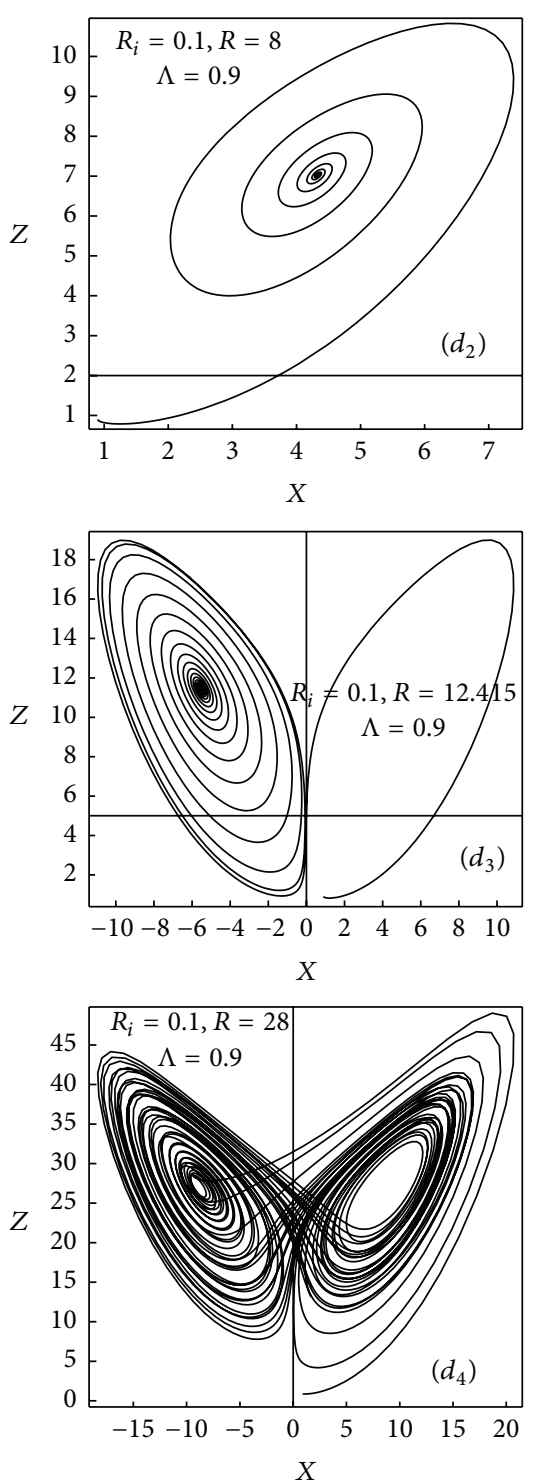

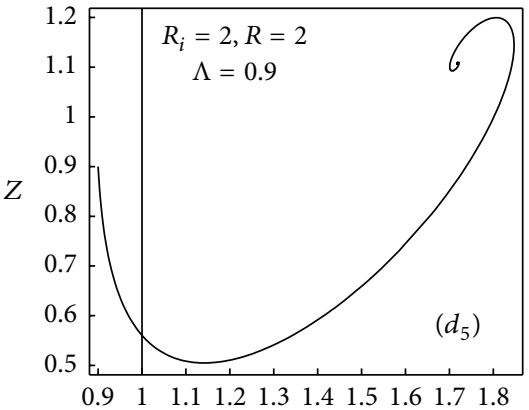

$X$
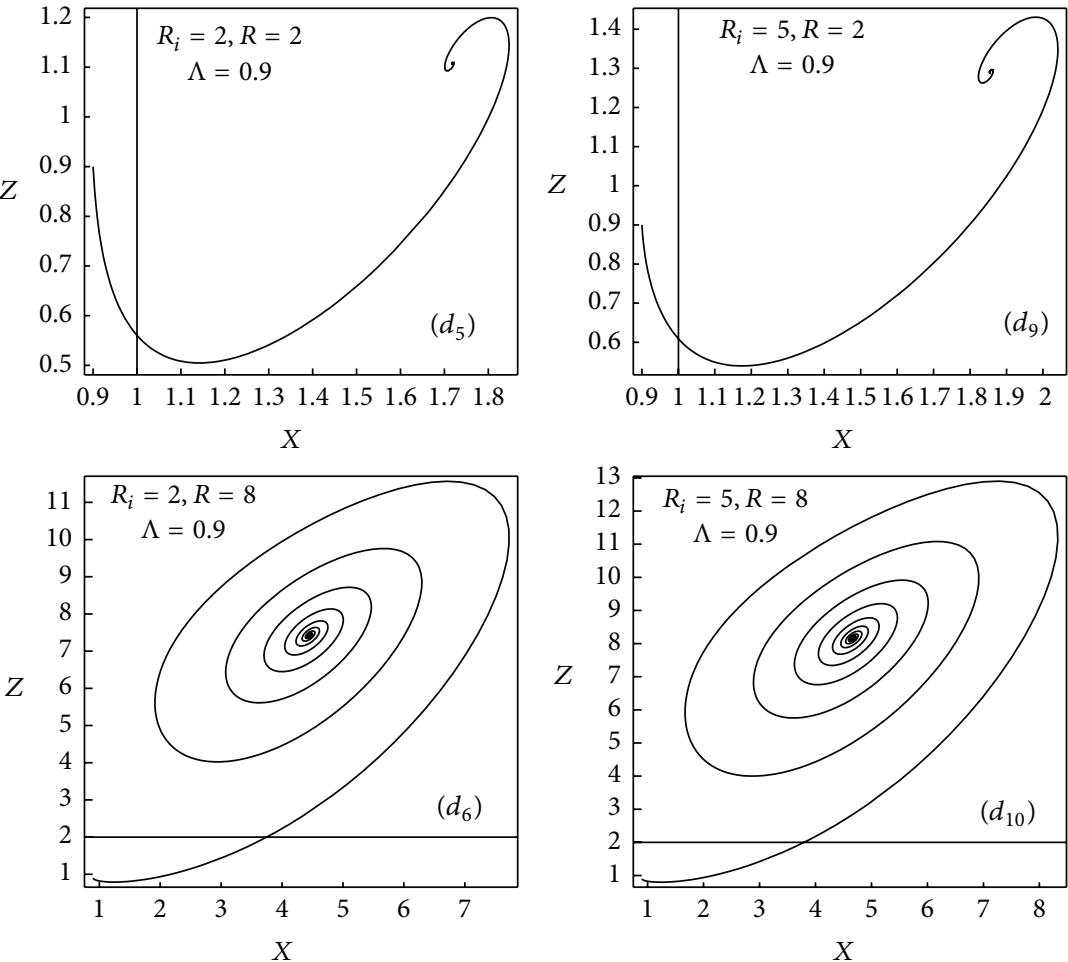

$X$
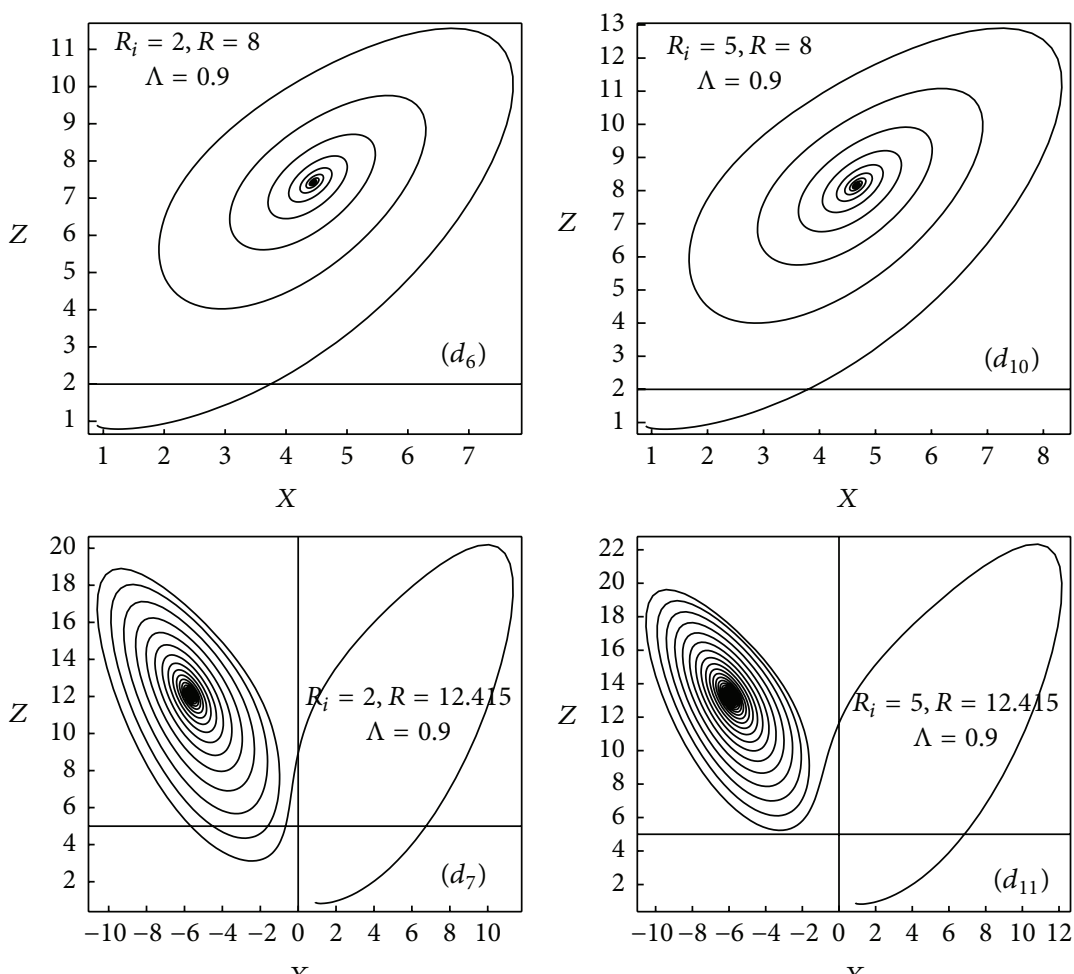

$X$
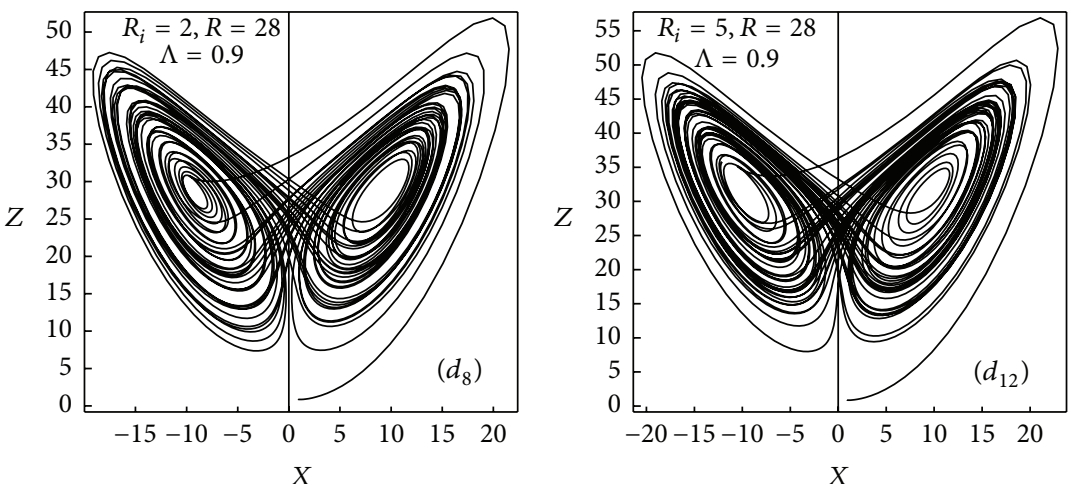

FIgURE 4: Projections and evolution of trajectories over the planes $X-Z$. 

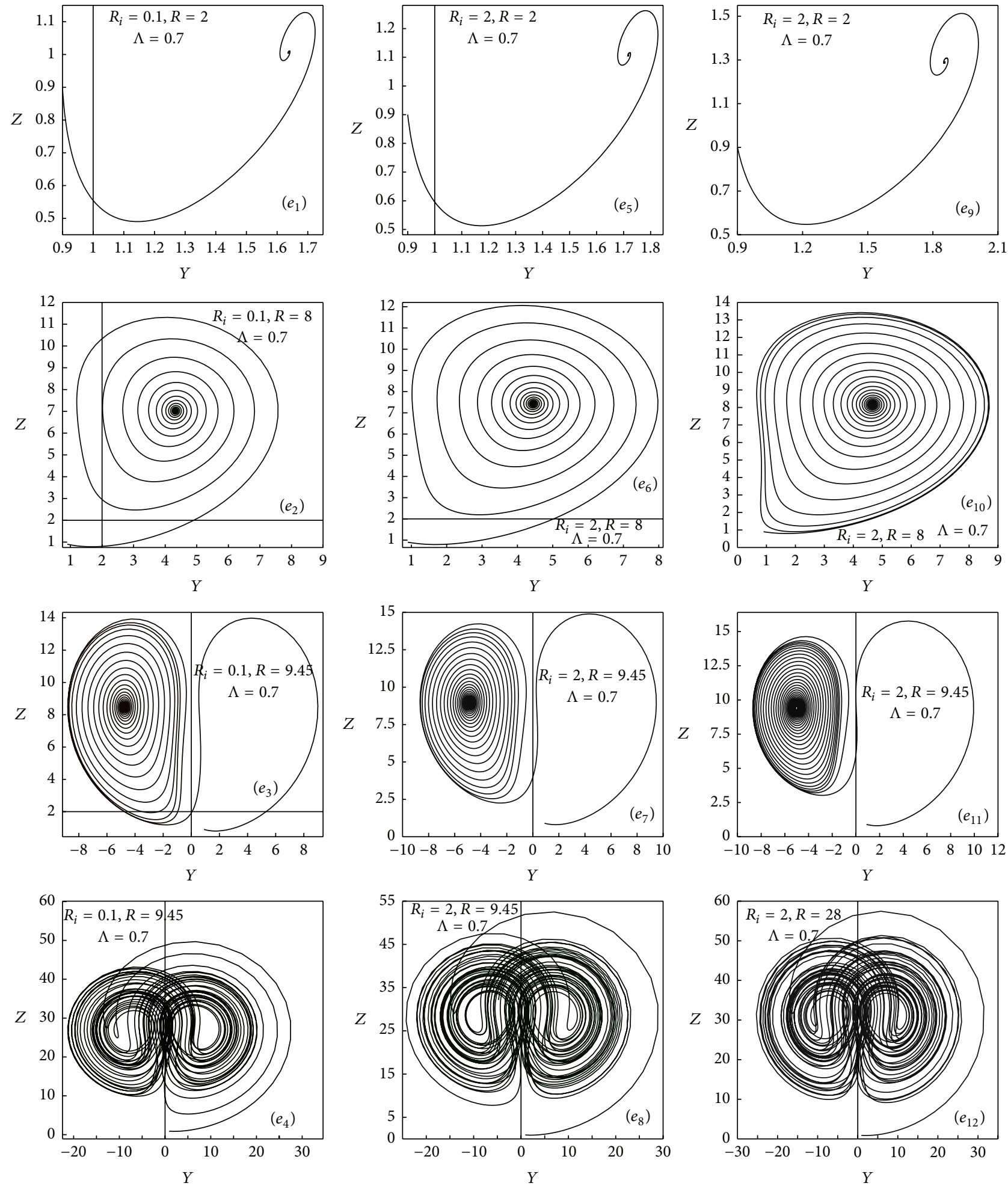

FIGURE 5: Projections and evolution of trajectories over the planes $Y-Z$. 

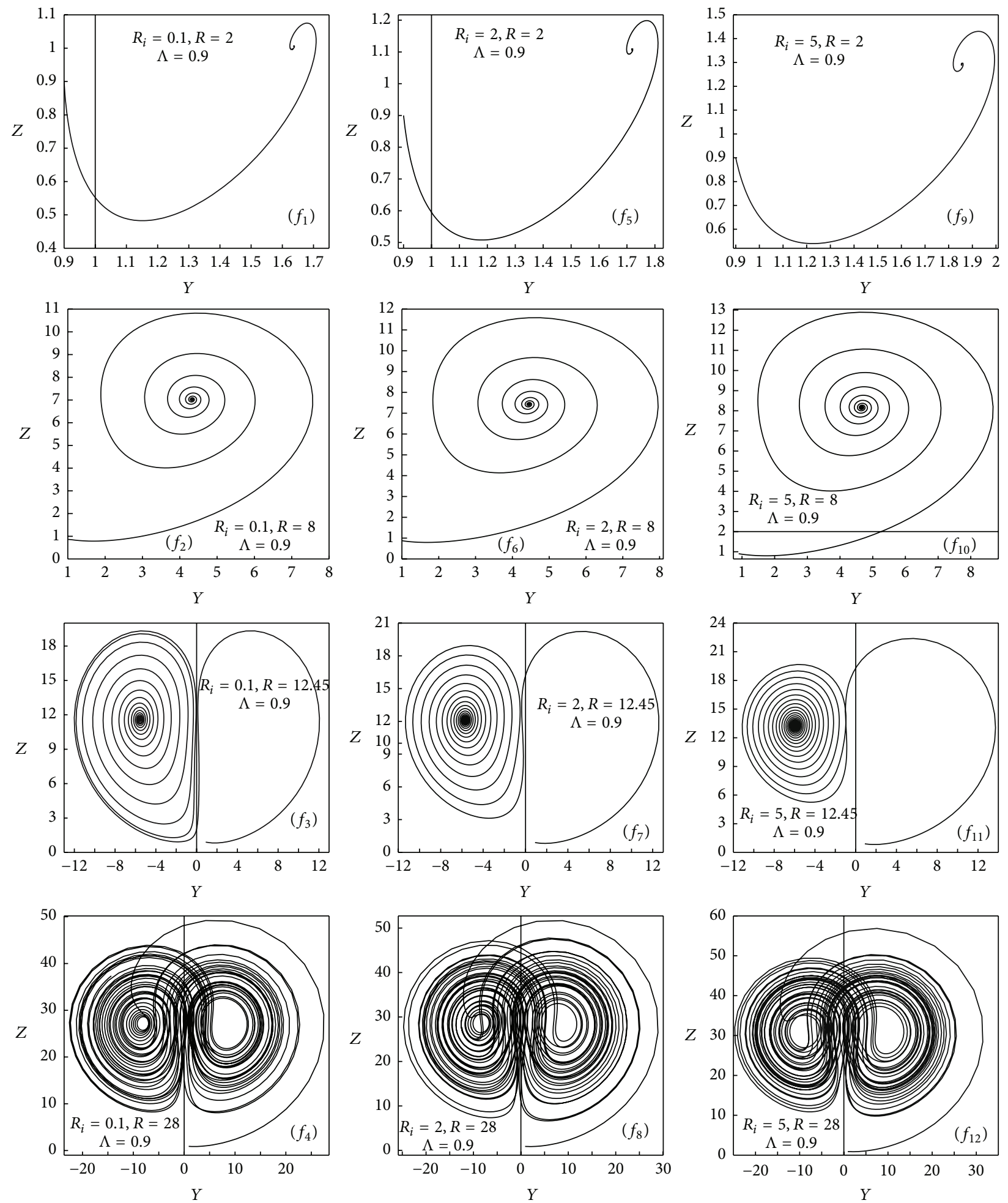

Figure 6: Projections and evolution of trajectories over the planes $Y-Z$. 

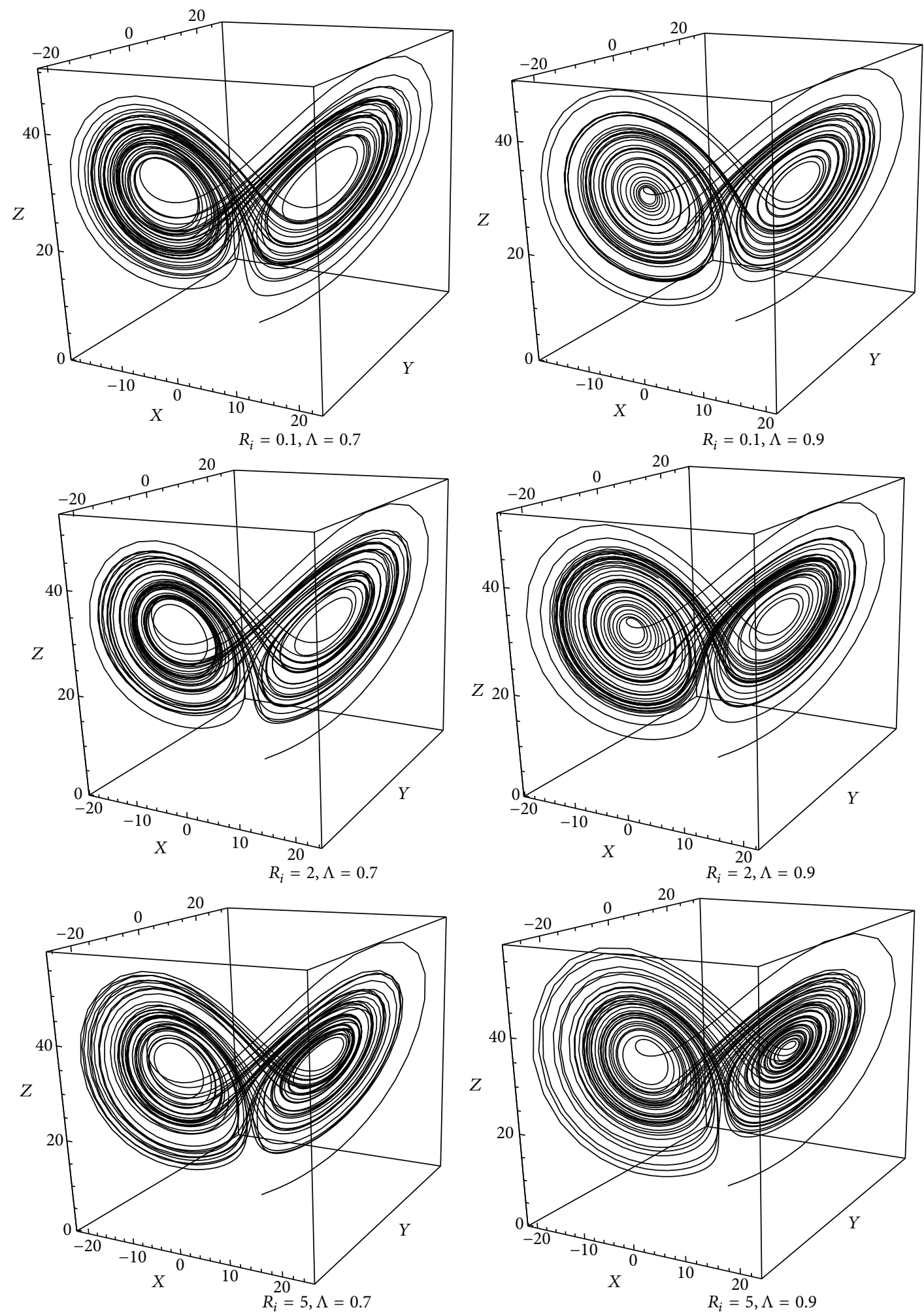

Figure 7: Projections and evolution of trajectories. 


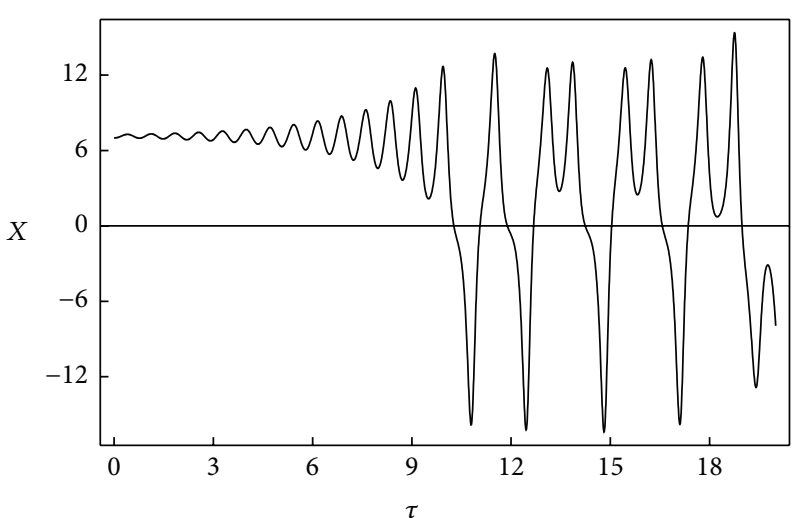

(a) $R_{i}=0.1, \sigma=10, b=-8 / 3$, and $R=20$; initial points $(7,7,19,0)$

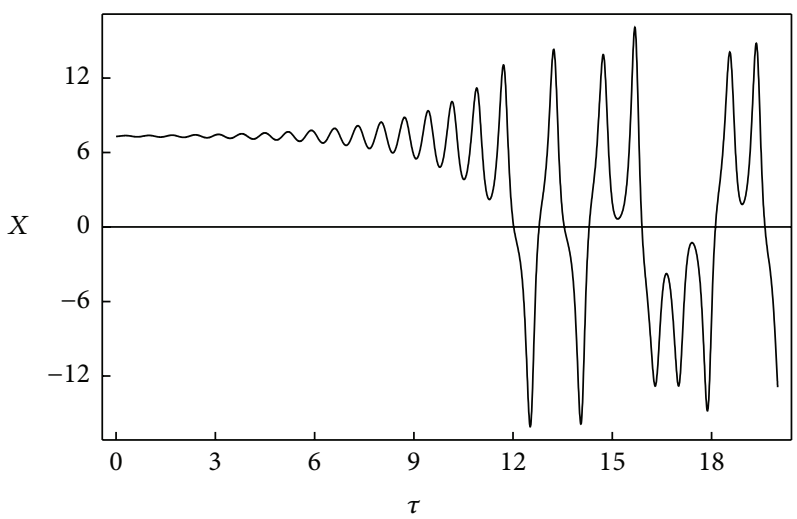

(c) $R_{i}=2, \sigma=10, b=-8 / 3$, and $R=20$; initial points $(7.3,7.3,20,0)$

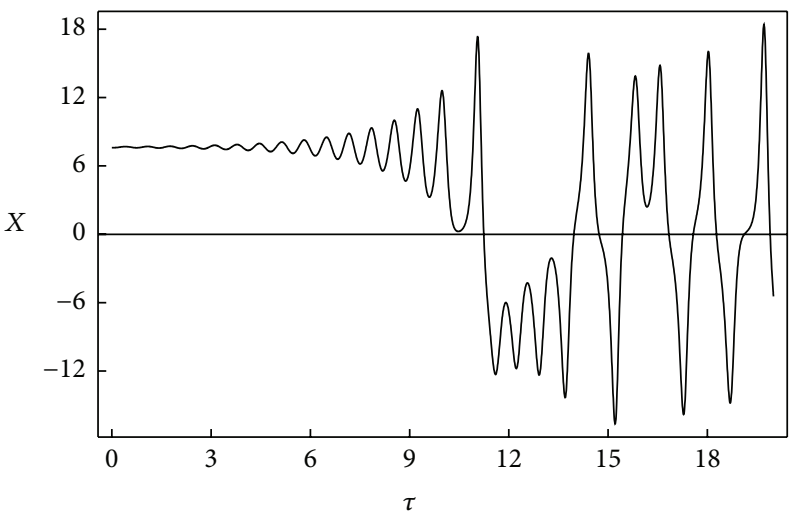

(e) $R_{i}=5, \sigma=10, b=-8 / 3$, and $R=20$; initial points $(7.6,7.6,21.9,0)$

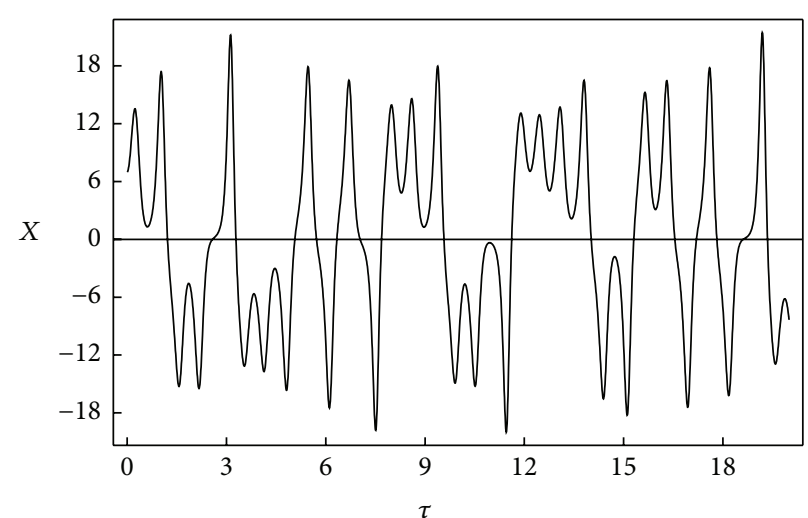

(b) $R_{i}=0.1, \sigma=10, b=-8 / 3$, and $R=28$; initial points $(7,7,19,0)$

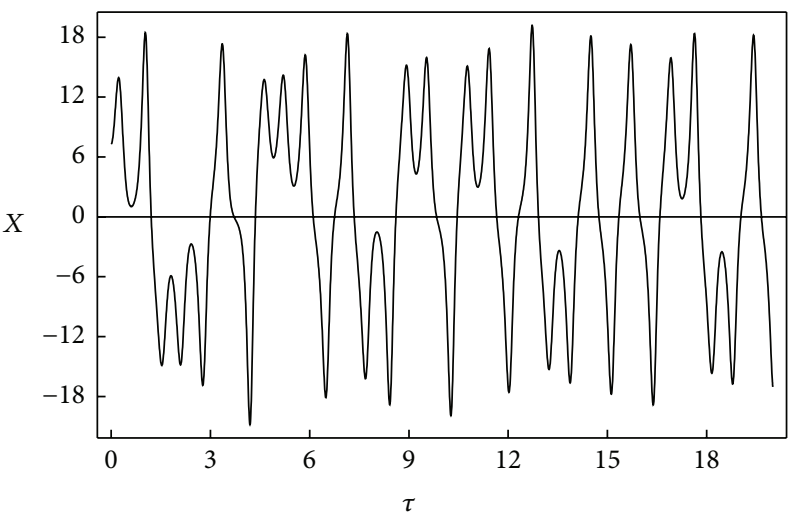

(d) $R_{i}=2, \sigma=10, b=-8 / 3$, and $R=28$; initial points $(7.3,7.3,20,0)$

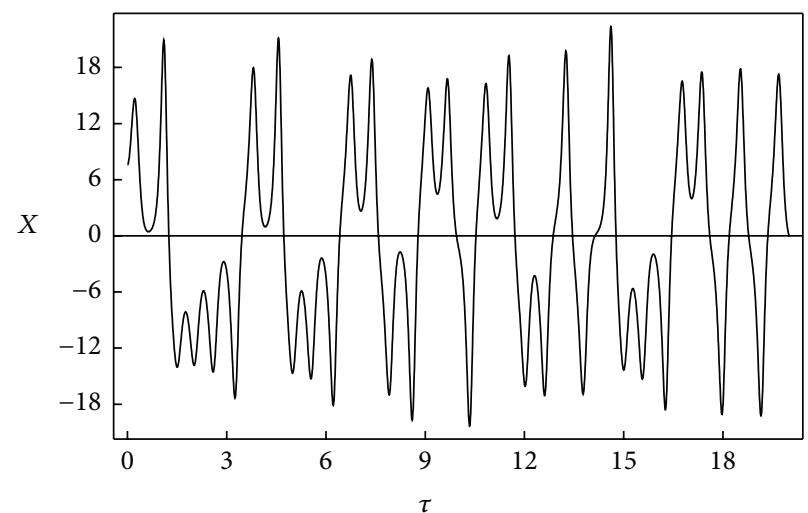

(f) $R_{i}=5, \sigma=10, b=-8 / 3$, and $R=28$; initial points $(7.6,7.6,21.9,0)$

FIGURE 8: The amplitude solution in the time domain $X(\tau)$.

time history of $Z$ at different values of $R_{i}$, at $R=20$ and $R=28$ with initial points $(7,7,19,0)$ for $R_{i}=0.1,(7.3,7.3,20,0)$ for $R_{i}=2$, and $(7.6,7.6,21.9,0)$ for $R_{i}=5$.

\section{Conclusions}

In this paper, chaotic convection in a viscoelastic fluid saturated porous medium with internal heat generation is analyzed. A four-dimensional system of differential equations is deduced by a Fourier series expansion. The effects of internal heat source and viscoelastic parameters are investigated on dynamics of convection. The following conclusions are drawn:

(1) An increment in the internal Rayleigh number accelerates the chaotic convection.

(2) Effect of increasing retardation time is to increase the amplitude of convection thus accelerating the chaotic convection.

(3) Effect of decreasing relaxation time is to accelerate the chaotic convection. 


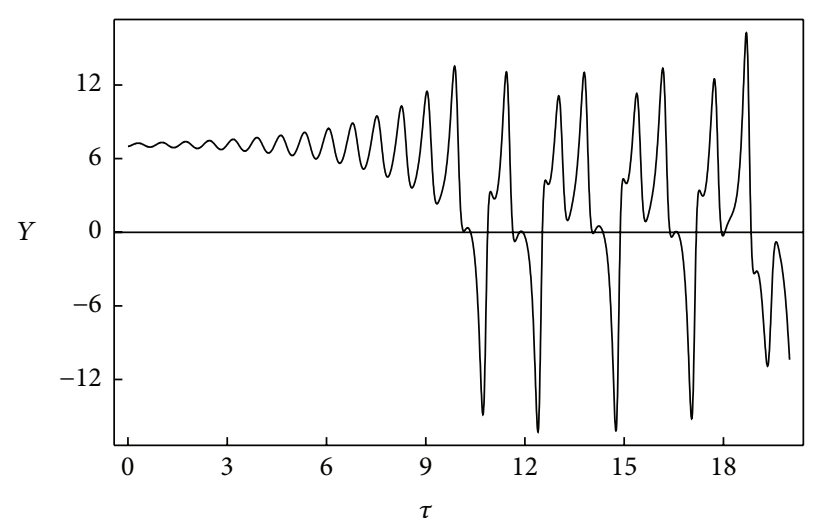

(a) $R_{i}=0.1, \sigma=10, b=-8 / 3$, and $R=20$; initial points $(7,7,19,0)$

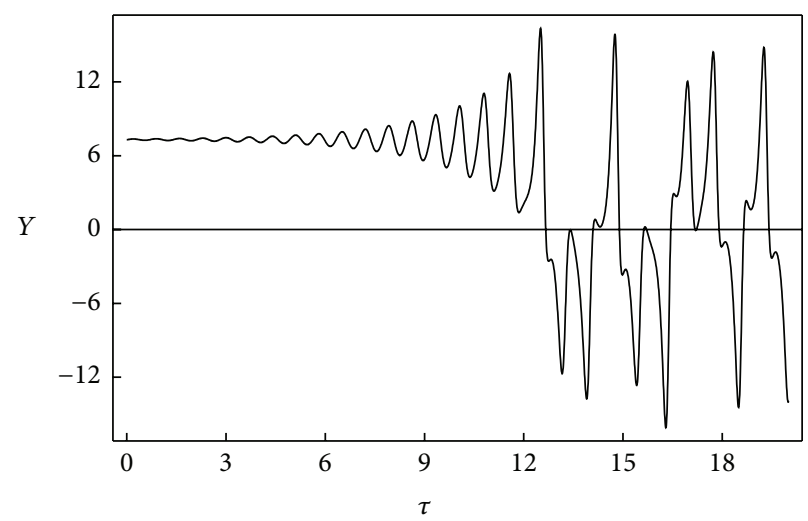

(c) $R_{i}=2, \sigma=10, b=-8 / 3$, and $R=20$; initial points $(7.3,7.3,20,0)$

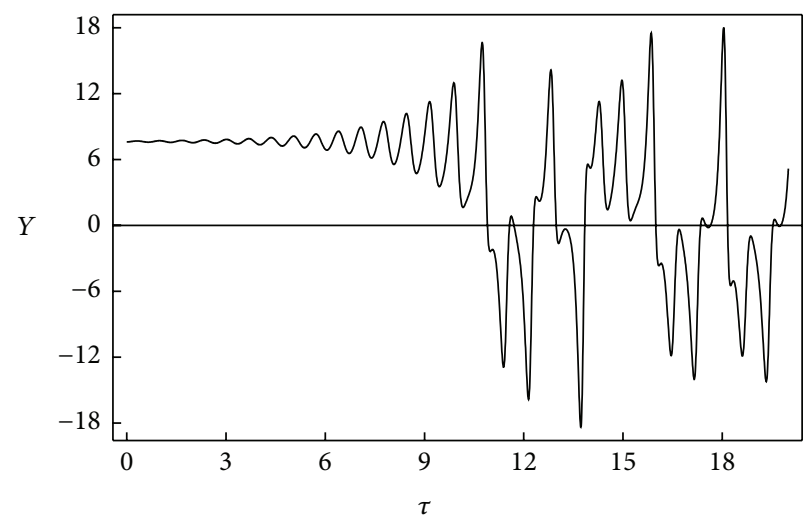

(e) $R_{i}=5, \sigma=10, b=-8 / 3$, and $R=20$; initial points $(7.6,7.6,21.9,0)$

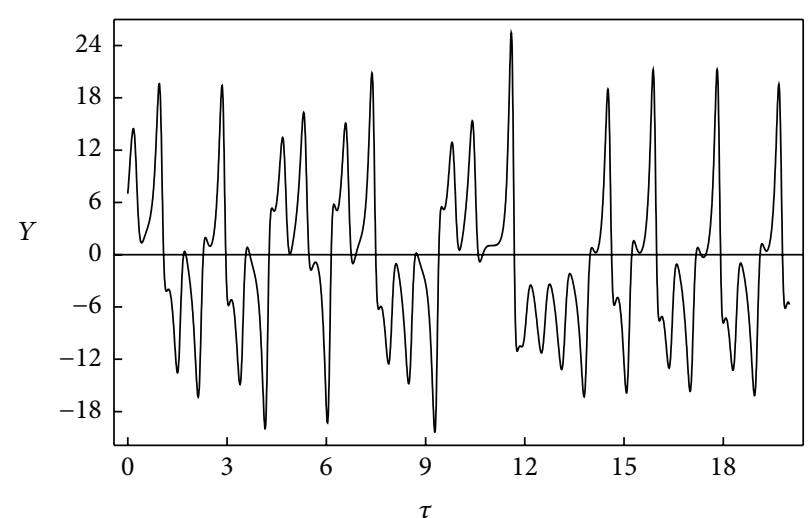

(b) $R_{i}=0.1, \sigma=10, b=-8 / 3$, and $R=28$; initial points $(7,7,19,0)$

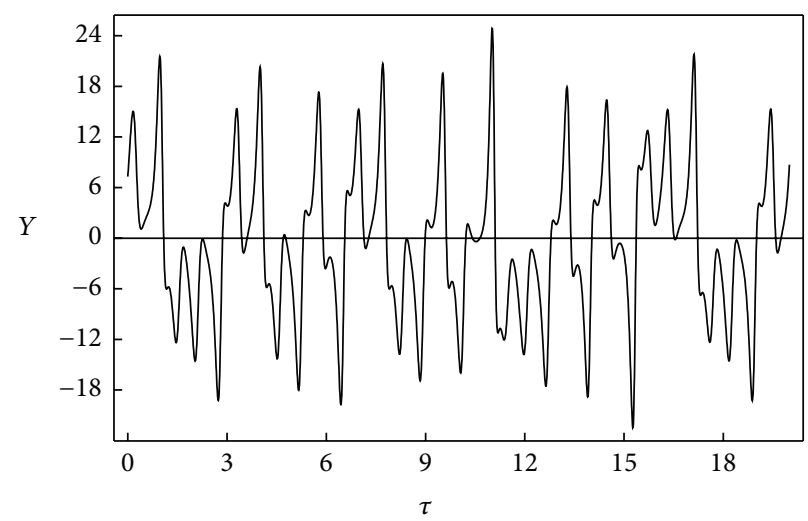

(d) $R_{i}=2, \sigma=10, b=-8 / 3$, and $R=28$; initial points $(7.3,7.3,20,0)$

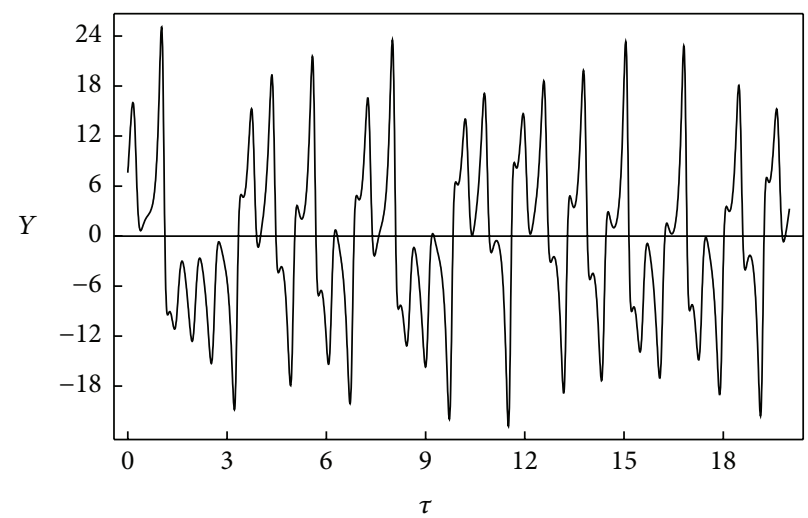

(f) $R_{i}=5, \sigma=10, b=-8 / 3$, and $R=28$; initial points $(7.6,7.6,21.9,0)$

FIGURE 9: The amplitude solution in the time domain $Y(\tau)$.

(4) As particular case, the present system reduces to the systems of Lorenz [9], Akhatov and Chembarisova [30], Sheu et al. [31], and Khayat [26-28].

\section{Nomenclature}

Latin Symbols
$a$ : Wave number
$a_{c}$ : Critical wave number
$d$ : Depth of the porous layer

Da: Darcy number, $K / d^{2}$

Pr: Prandtl number, $v / \kappa_{T}$

Va: Vadasz number, $\delta \operatorname{Pr} / \mathrm{Da}$

K: Permeability

$p:$ Pressure

q: $\quad$ Velocity $(u, v, w)$

Q: Internal heat source

Ra: Rayleigh number

$R_{i}$ : Internal Rayleigh number, $\left(Q d^{2} / \kappa_{T}\right)$

$t$ : Time

$T:$ Temperature 


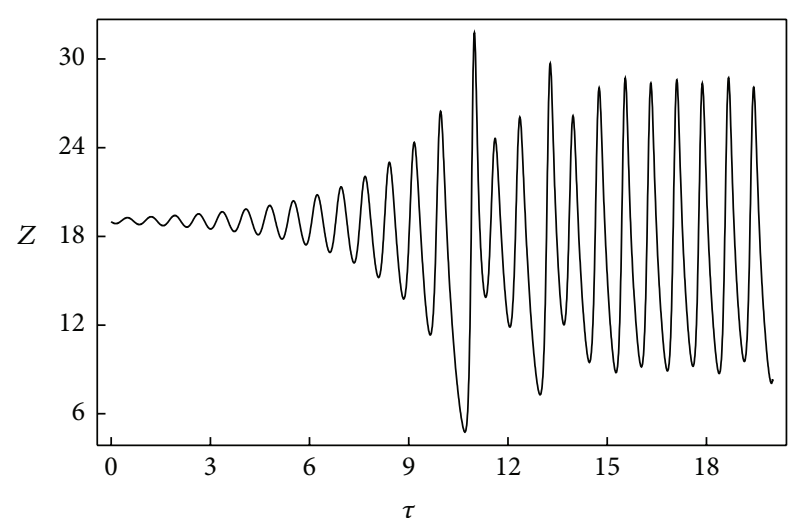

(a) $R_{i}=0.1, \sigma=10, b=-8 / 3$, and $R=20$; initial points $(7,7,19,0)$

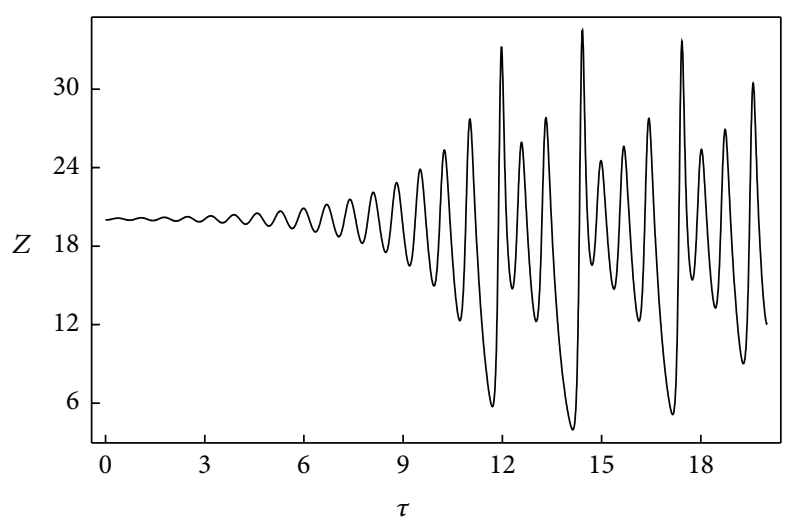

(c) $R_{i}=2, \sigma=10, b=-8 / 3$, and $R=20$; initial points $(7.3,7.3,20,0)$

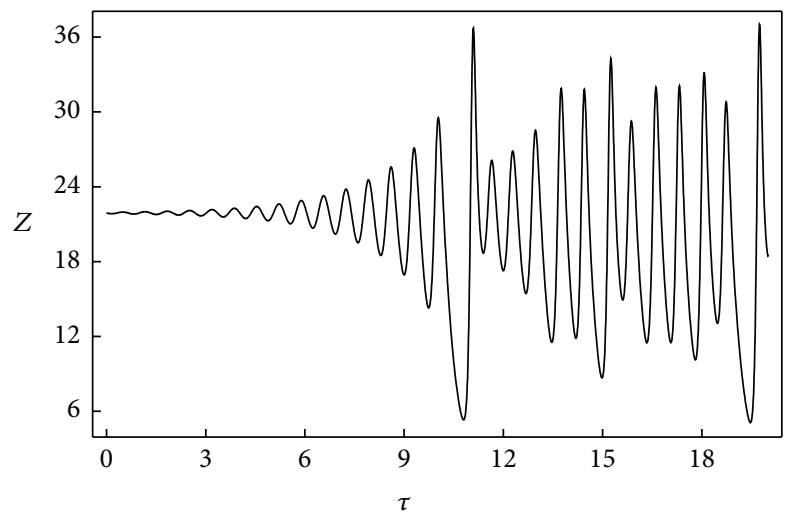

(e) $R_{i}=5, \sigma=10, b=-8 / 3$, and $R=20$; initial points $(7.6,7.6,21.9,0)$

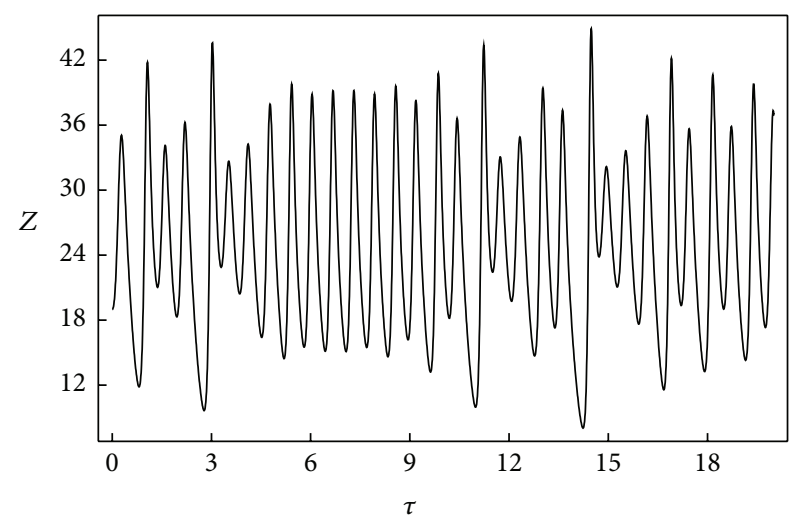

(b) $R_{i}=0.1, \sigma=10, b=-8 / 3$, and $R=28$; initial points $(7,7,19,0)$

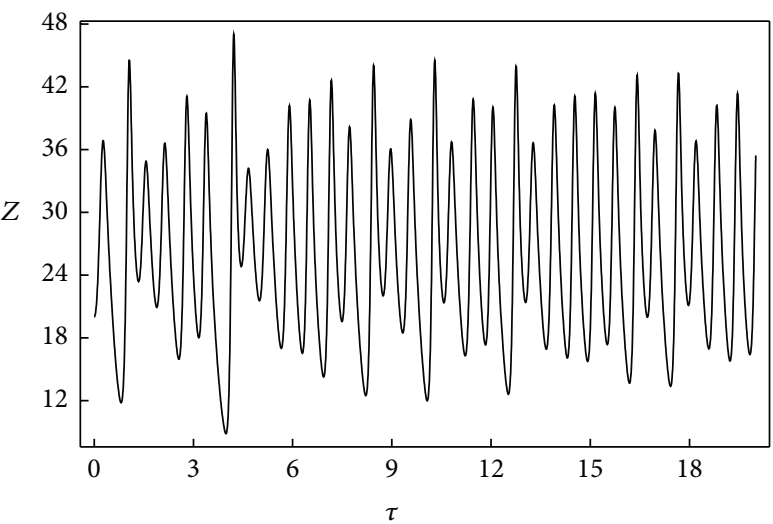

(d) $R_{i}=2, \sigma=10, b=-8 / 3$, and $R=28$; initial points $(7.3,7.3,20,0)$

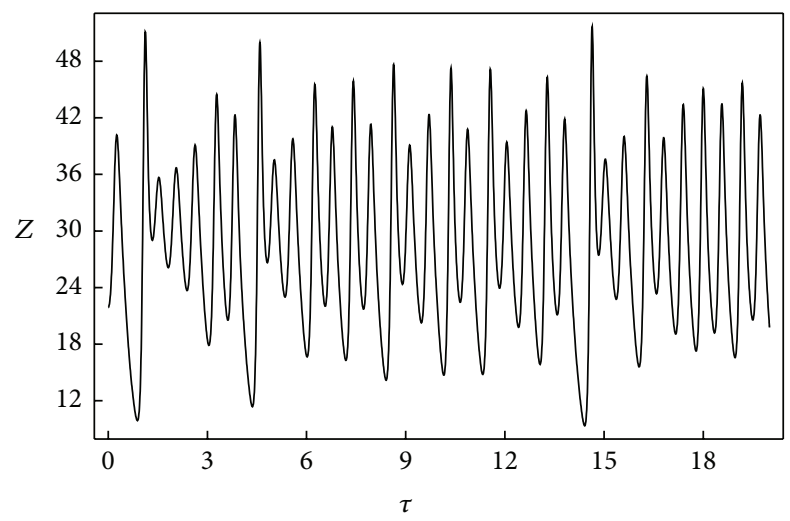

(f) $R_{i}=5, \sigma=10, b=-8 / 3$, and $R=28$; initial points $(7.6,7.6,21.9,0)$

FIgURE 10: The amplitude solution in the time domain $Z(\tau)$.

$\Delta T: \quad$ Temperature difference between the walls $(x, y, z)$ : Space coordinate.

\section{Greek Symbols}

$\lambda_{1}$ : Relaxation time

$\lambda_{2}$ : Retardation time

$\alpha_{T}$ : Thermal expansion coefficient

$\Gamma$ : Nondimensional relaxation time

$\Lambda$ : Ratio of retardation time to relaxation time, $\left(\lambda_{2} / \lambda_{1}\right)$

$\kappa_{T}$ : Thermal diffusivity $\phi$ : Porosity

$\rho$ : Density

$\mu$ : Dynamic viscosity

$\nu$ : Kinematic viscosity, $\mu / \rho_{0}$

$\psi$ : Stream function.

Other Symbols
$b$ : Basic state
c: Critical
0 : Reference state 
$\widehat{i}: \quad$ Unit normal vector in $x$-direction

$\hat{j}$ : Unit normal vector in $y$-direction

$\hat{k}$ : Unit normal vector in $z$-direction

$\nabla_{1}^{2}: \partial^{2} / \partial x^{2}+\partial^{2} / \partial y^{2}$, horizontal Laplacian

$\nabla^{2}: \nabla_{1}^{2}+\partial^{2} / \partial z^{2}$

$D: d / d z$.

\section{Conflict of Interests}

The author declares that there is no conflict of interests regarding the publication of this paper.

\section{References}

[1] C. W. Horton and F. T. Rogers, "Convection currents in a porous medium," Journal of Applied Physics, vol. 16, pp. 367-370, 1945.

[2] E. R. Lapwood, "Convection of a fluid in a porous medium," Mathematical Proceedings of the Cambridge Philosophical Society, vol. 44, no. 4, pp. 508-521, 1948.

[3] Y. Katto and T. Masuoka, "Criterion for the onset of convective flow in a fluid in a porous medium," International Journal of Heat and Mass Transfer, vol. 10, no. 3, pp. 297-309, 1967.

[4] D. B. Ingham and I. Pop, Transport Phenomena in Porous Media, vol. III, Elsevier, Oxford, UK, 2005.

[5] K. Vafai, Handbook of Porous Media, Taylor \& Francis, London, UK, CRC, Boca Raton, Fla, USA, 2006.

[6] D. A. Nield and A. Bejan, Convection in Porous Media, Springer, New York, NY, USA, 3rd edition, 2013.

[7] J. H. Poincaré, "Sur le problème des trois corps et les équations de la dynamique," Acta Mathematica, vol. 13, pp. 1-279, 1890.

[8] J. H. Poincaré, Les Méthodes Nouvelles de la Mécanique Céleste, Gauthier-Villars et Fils, Paris, France, 1899.

[9] E. N. Lorenz, "Deterministic non-periodic flow," Journal of the Atmospheric Sciences, vol. 20, no. 2, pp. 130-141, 1963.

[10] O. E. Rössler, "An equation for continuous chaos," Physics Letters A, vol. 57, no. 5, pp. 397-398, 1976.

[11] G. Chen and T. Ueta, "Yet another chaotic attractor," International Journal of Bifurcation and Chaos, vol. 9, no. 7, pp. 14651466, 1999.

[12] J. J. Vadász, J. P. Meyer, and G. Saneshan, "Chaotic and periodic natural convection for moderate and high Prandtl numbers in a porous layer subject to vibrations," Transport in Porous Media, vol. 103, no. 2, pp. 279-294, 2014.

[13] P. Kiran and B. S. Bhadauria, "Chaotic convection in a porous medium under temperature modulation," Transport in Porous Media, vol. 107, no. 3, pp. 745-763, 2015.

[14] B. Bhadauria, V. K. Gupta, I. Hasim, J. Jawdat, and A. Singh, "Chaotic convection in a rotating fluid layer," Alexandria Engineering Journal, vol. 54, no. 4, pp. 981-992, 2015.

[15] B. S. Bhadauria and P. Kiran, "Chaotic and oscillatory magnetoconvection in a binary viscoelastic fluid under g-jitter," International Journal of Heat and Mass Transfer, vol. 84, pp. 610-624, 2015.

[16] R. J. Marshall and A. B. Metzner, "Flow of viscoelastic fluids through porous media," Industrial and Engineering Chemistry Fundamentals, vol. 6, no. 3, pp. 393-400, 1967.

[17] D. F. James and D. R. McLaren, "The laminar flow of dilute polymer solutions through porous media," Journal of Fluid Mechanics, vol. 70, no. 4, pp. 733-752, 1975.

[18] M. C. Kim, S. B. Lee, S. Kim, and B. J. Chung, "Thermal instability of viscoelastic fluids in porous media," International
Journal of Heat and Mass Transfer, vol. 46, no. 26, pp. 50655072, 2003.

[19] D.-Y. Yoon, M. C. Kim, and C. K. Choi, "The onset of oscillatory convection in a horizontal porous layer saturated with viscoelastic liquid," Transport in Porous Media, vol. 55, no. 3, pp. 275-284, 2004.

[20] M. S. Malashetty, I. S. Shivakumara, S. Kulkarni, and M. Swamy, "Convective instability of Oldroyd-B fluid saturated porous layer heated from below using a thermal non-equilibrium model," Transport in Porous Media, vol. 64, no. 1, pp. 123-139, 2006.

[21] A. Kumar and B. S. Bhadauria, "Non-linear two dimensional double diffusive convection in a rotating porous layer saturated by a viscoelastic fluid," Transport in Porous Media, vol. 87, no. 1, pp. 229-250, 2011.

[22] A. Kumar and B. S. Bhadauria, "Thermal instability in a rotating anisotropic porous layer saturated by a viscoelastic fluid," International Journal of Non-Linear Mechanics, vol. 46, no. 1, pp. 47-56, 2011.

[23] A. Kumar and B. S. Bhadauria, "Double diffusive convection in a porous layer saturated with viscoelastic fluid using a thermal non-equilibrium model," Physics of Fluids, vol. 23, no. 5, Article ID 054101, 2011.

[24] B. S. Bhadauria and P. Kiran, "Heat and mass transfer for oscillatory convection in a binary viscoelastic fluid layer subjected to temperature modulation at the boundaries," International Communications in Heat and Mass Transfer, vol. 58, pp. 166-175, 2014.

[25] B. S. Bhadauria and P. Kiran, "Weakly nonlinear oscillatory convection in a viscoelastic fluid saturating porous medium under temperature modulation," International Journal of Heat and Mass Transfer, vol. 77, pp. 843-851, 2014.

[26] R. E. Khayat, "Chaos and overstability in the thermal convection of viscoelastic fluids," Journal of Non-Newtonian Fluid Mechanics, vol. 53, pp. 227-255, 1994.

[27] R. E. Khayat, "Fluid elasticity and the transition to chaos in thermal convection," Physical Review E, vol. 51, no. 1, pp. 380399, 1995.

[28] R. E. Khayat, "Non-linear overstability in the thermal convection of viscoelastic fluids," Journal of Non-Newtonian Fluid Mechanics, vol. 58, no. 2-3, pp. 331-356, 1995.

[29] E. Abu-Ramadan, J. M. Hay, and R. E. Khayat, "Characterization of chaotic thermal convection of viscoelastic fluids," Journal of Non-Newtonian Fluid Mechanics, vol. 115, no. 2-3, pp. 79-113, 2003.

[30] I. S. H. Akhatov and R. G. Chembarisova, "The thermo convective instability in hydrodynamics of relaxational liquids," in Instability in Multiphase Flows, G. Gouesbet and A. Berlemont, Eds., pp. 277-287, Plenum Press, New York, NY, USA, 1993.

[31] L.-J. Sheu, L.-M. Tam, J.-H. Chen, H.-K. Chen, K.-T. Lin, and Y. Kang, "Chaotic convection of viscoelastic fluids in porous media," Chaos, Solitons \& Fractals, vol. 37, no. 1, pp. 113-124, 2008.

[32] S. P. Bhattacharyya and S. K. Jena, "Thermal instability of a horizontal layer of micropolar fluid with heat source," Proceedings of the Indian Academy of Sciences-Mathematical Sciences, vol. 93, no. 1, pp. 13-26, 1984.

[33] M. Haajizadeh, A. F. Ozguc, and C. L. Tien, "Natural convection in a vertical porous enclosure with internal heat generation," International Journal of Heat and Mass Transfer, vol. 27, no. 10, pp. 1893-1902, 1984. 
[34] S. Rionero and B. Straughan, "Convection in a porous medium with internal heat source and variable gravity effects," International Journal of Engineering Science, vol. 28, no. 6, pp. 497-503, 1990.

[35] Y. F. Rao and B. X. Wang, "Natural convection in vertical porous enclosures with internal heat generation," International Journal of Heat and Mass Transfer, vol. 34, no. 1, pp. 247-252, 1991.

[36] C. Parthiban and P. R. Patil, "Thermal instability in an anisotropic porous medium with internal heat source and inclined temperature gradient," International Communications in Heat and Mass Transfer, vol. 24, no. 7, pp. 1049-1058, 1997.

[37] I. H. Herron, "Onset of convection in a porous medium with internal heat source and variable gravity," International Journal of Engineering Science, vol. 39, no. 2, pp. 201-208, 2001.

[38] A. Khalili and M. Huettel, "Effects of throughflow and internal heat generation on convective instabilities in an anisotropic porous layer," Journal of Porous Media, vol. 5, no. 3, pp. 64-75, 2002.

[39] M. V. Joshi, U. N. Gaitonde, and S. K. Mitra, "Analytical study of natural convection in a cavity with volumetric heat generation," Journal of Heat Transfer, vol. 128, no. 2, pp. 176-182, 2006.

[40] B. S. Bhadauria, A. Kumar, J. Kumar, N. C. Sacheti, and P. Chandran, "Natural convection in a rotating anisotropic porous layer with internal heat generation," Transport in Porous Media, vol. 90, no. 2, pp. 687-705, 2011.

[41] B. S. Bhadauria, "Double-diffusive convection in a saturated anisotropic porous layer with internal heat source," Transport in Porous Media, vol. 92, no. 2, pp. 299-320, 2012.

[42] B. S. Bhadauria, I. Hashim, and P. G. Siddheshwar, "Effect of internal-heating on weakly non-linear stability analysis of Rayleigh-Bénard convection under g-jitter," International Journal of Non-Linear Mechanics, vol. 54, pp. 35-42, 2013.

[43] E. A. Jackson, Perspectives of Nonlinear Dynamics, vol. 1-2, Cambridge University Press, 1991. 


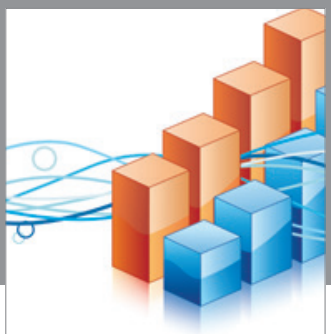

Advances in

Operations Research

vatem alat4

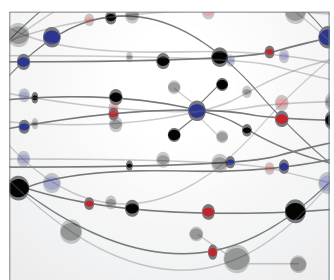

\section{The Scientific} World Journal
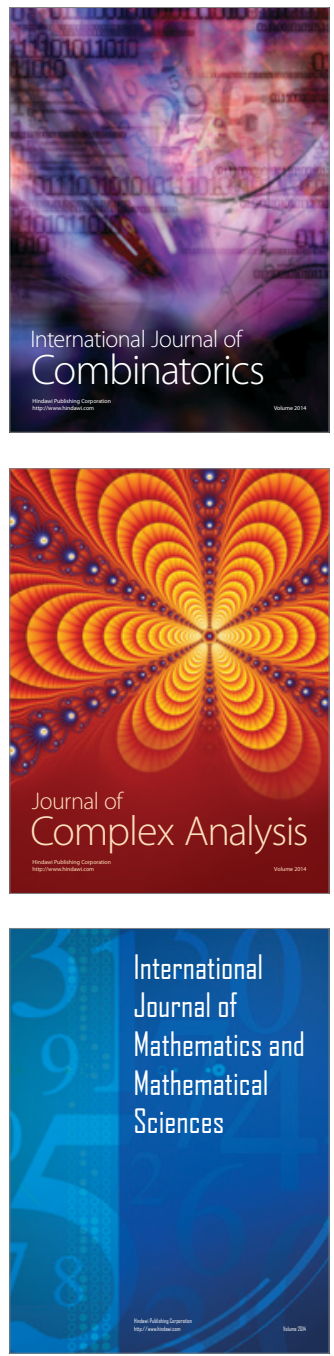
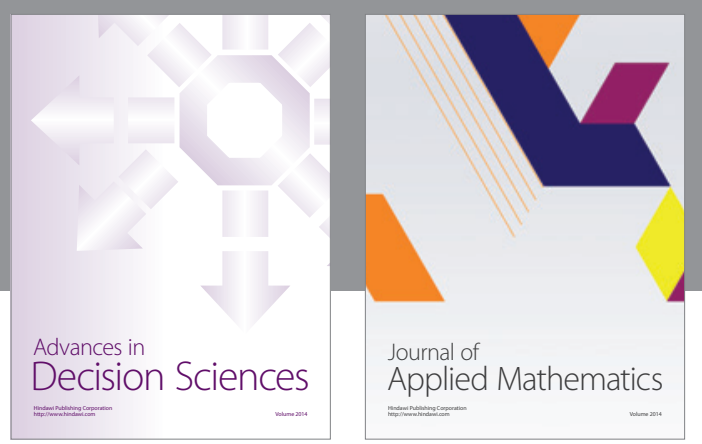

Algebra

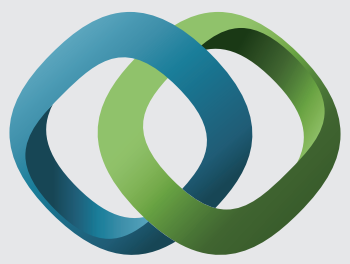

\section{Hindawi}

Submit your manuscripts at

http://www.hindawi.com
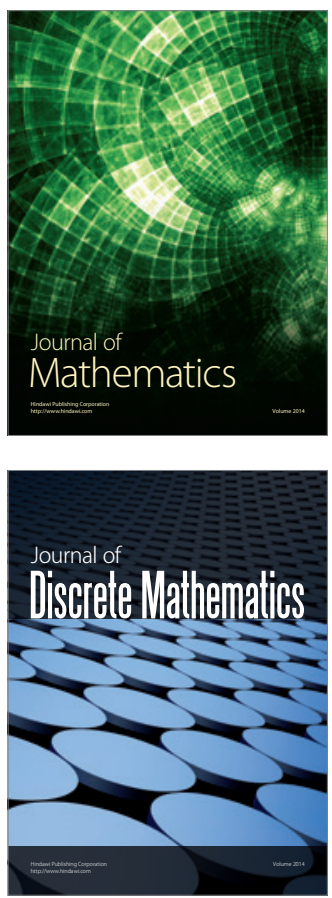

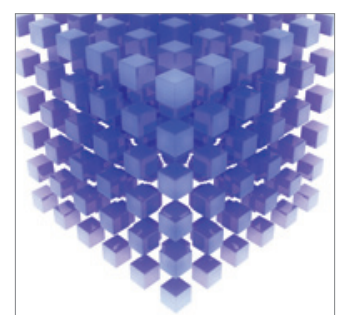

Mathematical Problems in Engineering
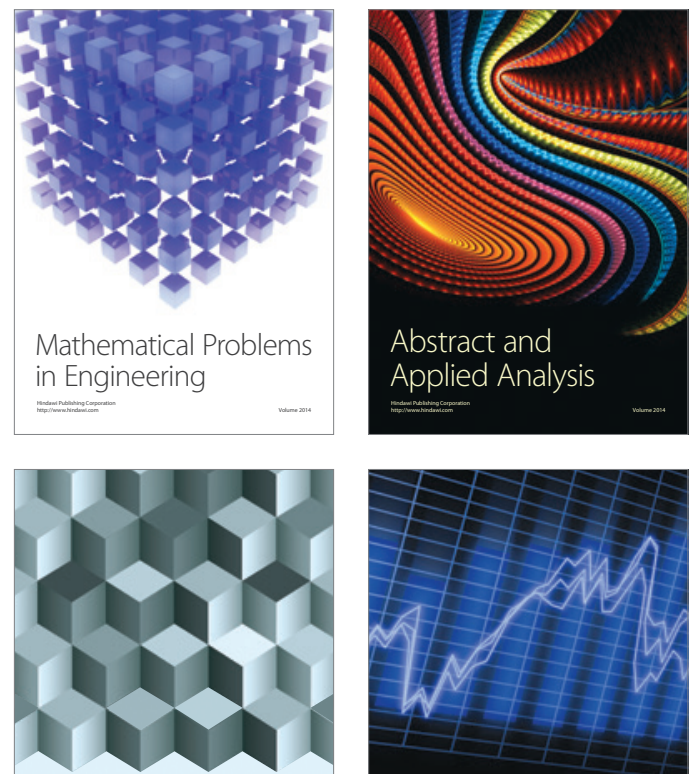

Journal of

Function Spaces

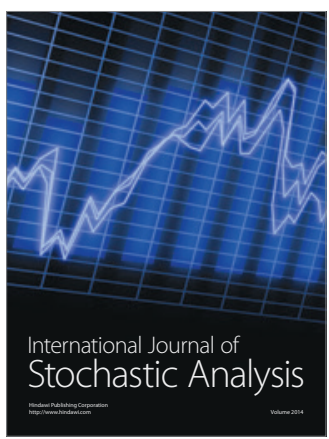

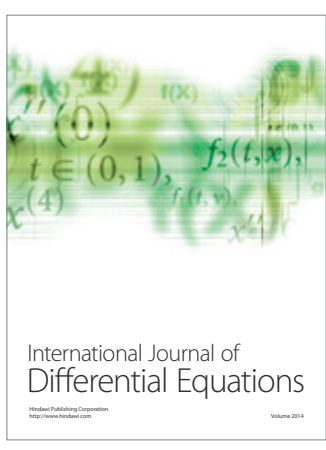
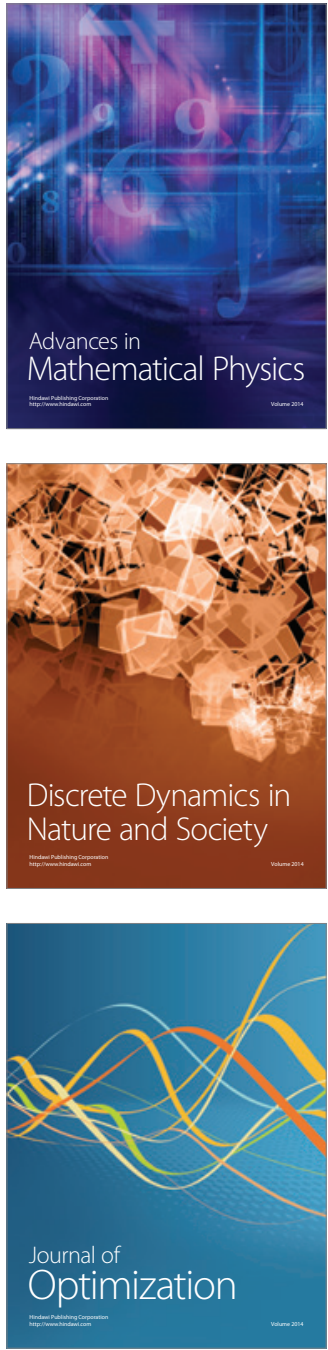